\title{
Structured sampling of olfactory input by the fly mushroom body
}

Zhihao Zheng ${ }^{1,2,3}$, Feng Li ${ }^{2}$, Corey Fisher ${ }^{2}$, Iqbal J. Ali ${ }^{2}$, Nadiya Sharifi' ${ }^{2}$, Steven Calle-Schuler ${ }^{2}$, Joseph $\mathrm{Hsu}^{2}$, Najla Masoodpanah ${ }^{2}$, Lucia Kmecova ${ }^{2}$, Tom Kazimiers ${ }^{2}$, Eric Perlman ${ }^{2,4}$, Matthew Nichols ${ }^{2}$, Peter H. Li ${ }^{5}$, Viren Jain ${ }^{5}$, Davi D. Bock ${ }^{2,6, *}$

${ }^{1}$ Princeton Neuroscience Institute, Princeton, New Jersey 08544, USA

${ }^{2}$ Howard Hughes Medical Institute Janelia Research Campus, 19700 Helix Drive, Ashburn, Virginia 20147, USA

${ }^{3}$ The Solomon H. Snyder Department of Neuroscience, The Johns Hopkins University, Baltimore, MD, USA

${ }^{4}$ Yikes LLC, Baltimore, MD, USA

${ }^{5}$ Google Research, Mountain View, CA, USA

${ }^{6}$ Department of Neurological Sciences, University of Vermont, Burlington, VT, USA

*To whom correspondence should be addressed.

\section{Abstract}

Associative memory formation and recall in the adult fruit fly Drosophila melanogaster is subserved by the mushroom body (MB). Upon arrival in the MB, sensory information undergoes a profound transformation. Olfactory projection neurons (PNs), the main MB input, exhibit broadly tuned, sustained, and stereotyped responses to odorants; in contrast, their postsynaptic targets in the $\mathrm{MB}$, the Kenyon cells (KCs), are nonstereotyped, narrowly tuned, and only briefly responsive to odorants. Theory and experiment have suggested that this transformation is implemented by random connectivity between KCs and PNs. However, this hypothesis has been challenging to test, given the difficulty of mapping synaptic connections between large numbers of neurons to achieve a unified view of neuronal network structure. Here we used a recent whole-brain electron microscopy (EM) volume of the adult fruit fly to map large numbers of PNto-KC connections at synaptic resolution. Comparison of the observed connectome to precisely defined null models revealed unexpected network structure, in which a subset of food-responsive 
PN types converge on individual downstream KCs more frequently than expected. The connectivity bias is consistent with the neurogeometry: axons of the overconvergent PNs tend to arborize near one another in the MB main calyx, making local $\mathrm{KC}$ dendrites more likely to receive input from those types. Computational modeling of the observed PN-to-KC network showed that input from the overconvergent PN types is better discriminated than input from other types. These results suggest an 'associative fovea' for olfaction, in that the MB is wired to better discriminate more frequently occurring and ethologically relevant combinations of foodrelated odors.

\section{Introduction}

The cellular basis for associative memory formation and recall remains a central mystery of neurobiology. Connectomics, in which synaptic connections are traced between large numbers of neurons to map circuit wiring diagrams (Lichtman and Sanes, 2008), offers a new method by which to explore the topic. Given the current capabilities of electron microscopy (EM)-based connectomics technologies (Kornfeld and Denk, 2018), the adult fruit fly Drosophila melanogaster is arguably an ideal model system for investigating the neuronal networks underpinning learning and memory. Its brain is small enough to have been completely imaged at synaptic resolution by electron microscopy (Zheng et al., 2018); it is behaviorally sophisticated (DasGupta et al., 2014; Dickinson and Muijres, 2016; Ofstad et al., 2011; Owald and Waddell, 2015); and the stereotyped morphology and physiology of its cell types allow ready integration of information across individuals (Costa et al., 2016; Nern et al., 2015). Each cell type normally consists of one or a handful of neurons (Aso et al., 2014; Meinertzhagen, 2010; Scheffer et al., 2020), which may be individually addressed using genetic tools, allowing circuits to be 
functionally imaged and perturbed in a highly specific fashion (Dana et al., 2016; Dionne et al., 2018; Klapoetke et al., 2014; Venken et al., 2011).

The exception to this norm is the mushroom body (MB; Figure 1A), a bilaterally symmetric structure for associative memory formation and recall (Groschner and Miesenbock, 2019; Guven-Ozkan and Davis, 2014; Heisenberg, 2003). The MB contains about 2,200 intrinsic neurons, called Kenyon cells (KCs), on each side of the fly brain (Aso et al., 2009; Bates et al., 2020; Technau and Heisenberg, 1982). Kenyon cells can be divided into three main subtypes, $\gamma$, $\alpha^{\prime} / \beta^{\prime}, \alpha / \beta$ (Crittenden et al., 1998; Lee et al., 1999; Tanaka et al., 2008), and the axons of each subtype project to the eponymous lobe where the KCs provide input to a relatively small number of MB output neurons (21 cell types comprising 34 neurons, Aso et al., 2014; Aso and Rubin, 2020). Sensory afferents to KCs are dominated by 150 olfactory projection neurons (PNs), which relay information from the 51 olfactory glomeruli of the antennal lobe (AL; Bates et al., 2020; Jefferis et al., 2007; Stocker et al., 1990; Wong et al., 2002). Projection neuron morphology and odorant response profiles are highly stereotyped across individuals, and exhibit broad tuning and sustained responses to panels of odorants (Bhandawat et al., 2007; Costa et al., 2016). Olfactory PNs project to the rear of the brain and collateralize in the MB main calyx, providing input to $\mathrm{KC}$ dendrites. Each $\mathrm{KC}$ dendrite terminates in specialized 'claws', each of which ensheathes a single PN axonal bouton (Figure 1B). Multiple KC claws commonly ensheath a given PN bouton, and each KC samples input from an average of $\sim 6-8$ PNs (Butcher et al., 2012; Caron et al., 2013; Leiss et al., 2009; Yasuyama et al., 2002). Multiple input PNs must be coactive in order to evoke an action potential in a given $\mathrm{KC}$ (Gruntman and Turner, 2013), and widefield feedback inhibition is preponderant throughout the MB (Lin et al., 2014), 
resulting in $\mathrm{KC}$ activity that is much sparser and more sharply tuned than PNs (Turner et al., 2008).

The PN-to-KC layer therefore implements a transformation of olfactory representation: broad, stereotyped, and sustained olfactory responses, in a small population of PNs, are converted to sparse, variable, and transient responses, distributed across a large population of KCs. This circuit architecture is an example of a 'Marr motif' (Litwin-Kumar et al., 2017; Stevens, 2015), after the theorist David Marr's foundational work on cerebellar function (Albus, 1971; Marr, 1969). The Marr motif is found in brain regions from different animal species, including cerebellum, hippocampus, and piriform cortex in vertebrates, and even the vertical lobe of the octopus (Cayco-Gajic and Silver, 2019; Farris, 2011; Shomrat et al., 2015; Stevens, 2015). In the fly, it is thought to permit efficient representation of arbitrary combinations of odorants - which may be thought of as points in a high-dimensional olfactory space - for downstream use as a conditioned stimulus during associative memory formation and recall (Cayco-Gajic and Silver, 2019; Groschner and Miesenbock, 2019; Perisse et al., 2013). Theoretical analyses have argued that randomly mixing different input channels, when combined with a nonlinearity such as a spike threshold, increases the dimensionality, and, therefore, the linear separability of activity patterns, making them easier to discriminate (Babadi and Sompolinsky, 2014; Barak et al., 2013; Hansel and van Vreeswijk, 2012; Rigotti et al., 2013). Most models of the PN-to-KC network in the fly have therefore assumed that in the Marr motif, input neurons (PNs) connect to the intrinsic neurons (KCs) at random (Dasgupta et al., 2017; Eichler et al., 2017; Litwin-Kumar et al., 2017; Stevens, 2015; but see Koulakov et al., 2011; Pehlevan et al., 2017).

Several substantial efforts to test the hypothesis of random PN-to-KC connectivity have been made. In the fruit fly larva, a complete PN-to-KC connectome was mapped using a whole-CNS 
electron microscopy (EM) volume (Eichler et al., 2017). No evidence of network structure was found, although single claw KCs were found to occur more frequently than a gaussian distribution would predict. However, the larval MB is qualitatively and quantitatively different from that of the adult, in that it contains only about $100 \mathrm{KCs}$ per hemisphere (all of which are of a single class $\gamma$; Lee et al., 1999). In adult flies, single-cell retrograde labeling was used to identify the PN inputs to a single KC in each of 200 individual flies (Caron et al., 2013). About half the claws for each $\mathrm{KC}$ were successfully labeled. No evidence of network structure was found, although some PN types clearly had more downstream targets than others. Finally, electrophysiological recordings of $23 \mathrm{KCs}$ across 27 adult fruit flies revealed highly diverse olfactory responses, with only two KCs exhibiting an identical response profile across individuals (Murthy et al., 2008). Overall, the relatively small sample sizes of the adult datasets have sufficed to exclude highly structured PN-to-KC connectivity graphs, but have not proved randomness.

Indeed, several studies have hinted at the existence of PN-to-KC network structure.

Anatomically, PN axonal arbors and $\mathrm{KC}$ dendritic arbors are known to occupy stereotyped positions within the MB calyx as a function of cellular subtype (Jefferis et al., 2007; Lin et al., 2007; Tanaka et al., 2004; Zheng et al., 2018). Physiologically, calcium imaging has revealed that $\mathrm{KC}$ claws show more correlated responses than would be predicted by chance, and simultaneous optogenetic stimulation of three PN subtypes (comprising 13 PNs in total) also showed greater-than-chance convergence (Gruntman and Turner, 2013).

Whether the PN-to-KC network is fully random, or has some structure, therefore is an open question. To address it we surveyed a large number of PN-to-KC connections, using the previously described Female Adult Fly Brain (“FAFB") EM volume (Zheng et al., 2018). The 
resulting sample of this Marr motif had far greater statistical power than previously obtained datasets, allowing previously undetected network structure to be revealed.

\section{Results}

To map the PN-to-KC network, KCs were randomly selected for reconstruction from a crosssection of the $\mathrm{MB}$ pedunculus, a tract where $\mathrm{KC}$ axons converge after their dendrites receive input in the MB main calyx (Figure 1A-B; Supplemental Figure 1A-C). The PN bouton innervating each $\mathrm{KC}$ claw was then retrogradely traced to the main PN axon trunk, and the PN type was identified, using previously published classifications of PNs in the FAFB dataset (Zheng et al., 2018). Initially, reconstructions were traced purely manually; later reconstructions leveraged an automated segmentation of the full FAFB dataset (Li et al., 2019). All olfactory PN input to 7,102 claws arising from $1,356 \mathrm{KCs}$ was mapped and identified ( $\sim 62 \%$ of all claws on the right side of the brain; $440 \mathrm{KCs}$ were manually traced, and 916 were reconstructed using automated segmentation). Consistent with previous studies (Butcher et al., 2012; Caron et al., 2013), each reconstructed $\mathrm{KC}$ was found to have 5.2 claws on average (Supplemental Figure 2A). The number of KCs postsynaptic to each PN subtype was also in excellent agreement with counts obtained from a recently released connectome of adult fly brain connectivity (Supplemental Figure 2B-C; Scheffer et al., 2020). The consistency of these metrics across datasets and methods indicates that the PN-to-KC network reconstructed in the present study is of high quality and therefore suitable for detailed analysis.

If $\mathrm{PN}-$ to-KC connectivity were random, the probability that a $\mathrm{KC}$ receives input from one $\mathrm{PN}$ type is, by definition, independent from whether it gets input from any other type. To test whether these input probabilities are in fact independent, several null models were tested. In the 
first, the "random bouton" model, a large number of randomized PN-to-KC maps were generated, wherein each claw of each reconstructed $\mathrm{KC}$ was assigned a PN bouton selected (with replacement) at random. For each $\mathrm{KC}$, the expected distribution of the number of inputs from each PN type in the random bouton model was obtained. Then, a conditional input analysis was performed, to determine whether KCs are more or less likely than expected to get input from a particular PN type (Figure 1C, matrix columns), given input from another PN type (Figure 1C, matrix rows). Conditional probabilities were quantified as z-scores (the number of standard deviations of the observed value from the mean of the null distribution).

Unsupervised clustering of conditional input probabilities revealed a distinctive 'community' of PN types which converge onto KCs at above-chance levels (Figure 1D, PN types in bold). The mean community z score was significantly higher (Supplemental Figure $3 \mathrm{~A} ; 5.7 \pm 2.9$ ) than noncommunity PN combinations (Supplemental Figure 3C; $-0.5 \pm 1.5$; Supplemental Figure 3A vs. $\left.3 \mathrm{C}, \mathrm{p}<1 \times 10^{-8}\right)$. Additional PN combinations also showed elevated z-scores, but mean z-score for these was significantly lower than the selected subset comprising the community (Supplemental Figure 3B; $2.3 \pm 1.9$; Supplemental Figure 3A vs. 3B, $\mathrm{p}<1 \times 10^{-8}$ ). Analysis of individual randomized maps of $\mathrm{PN}$-to-KC connectivity revealed no such clustering (Supplemental Figure 3E). Similar results were obtained using covariance analysis (Supplemental Figure 4).

Following identification of the overconvergent PN community, a literature review was conducted to determine the broad categories of odorants each PN type responds to. Strikingly, all PN types within the community were found to respond preferentially to food-related odorants (Figure 1D; Badel et al., 2016; Hallem and Carlson, 2006; Laissue and Vosshall, 2008; Mansourian and Stensmyr, 2015; Root et al., 2007; Schubert et al., 2014; Semmelhack and Wang, 2009), 
suggesting that the observed $\mathrm{PN}$-to-KC network structure might play a distinctive role in $\mathrm{MB}$ circuit function. Although the MB main calyx contains a great deal of recurrent circuitry (Butcher et al., 2012; Christiansen et al., 2011), with some cell types that are as yet little understood (Zheng et al., 2018), a simplifying feed-forward model of the PN-to-KC network has previously been used to study its performance on classification tasks (Eichler et al., 2017; Litwin-Kumar et al., 2017). When this model was modified to incorporate the observed PN-to$\mathrm{KC}$ network structure, increased activation of community PNs was found to improve classification performance (Figure 1E-F; Supplemental Figure 5). Increased activation of all food-preferring PNs, which includes PN types in addition to the overconvergent community PNs, also led to superior classification performance (Supplemental Figure 5A).

To determine how over-convergence by community PNs is generated, the underlying neuronal network anatomy was further analyzed. Community PN boutons are ensheathed by many more $\mathrm{KC}$ claws than expected from the random bouton model (Figure 2A-B). Conversely, fewer KCs than predicted by the random bouton model receive input from the community PN types (Figure 2C). This suggested that the observed network structure might result simply from more ensheathment of community PN boutons by KC claws. To test this hypothesis, a second null model was devised, in which each bouton selects a claw at random (without replacement), and the number of claws ensheathing each bouton is held equal to the observed value. In this "random claw" model, both the number of inputs to each $\mathrm{KC}$ and the number of outputs from each PN type are held constant. Clustering of z-scores of the observed PN-to-KC connectivity using the random claw null model revealed the same group of community PNs, albeit with lower variance (Figure 2D; Supplemental Figure 6A-B). Although the random claw model captured 
more of the observed network structure, over-sampling of the community PNs by KCs (Figure 2A) alone is therefore insufficient to explain the community cluster.

In contrast, application of these analysis methods to an earlier sampling of PN-to-KC connectivity (Caron et al., 2013) failed to reveal the community of overconvergent PNs (Supplemental Figure 7A-C). However, that study mapped many fewer PN-to-KC connections (about half the claws in each of $200 \mathrm{KCs} ; 1 \mathrm{KC}$ mapped per fly). When the data generated in the present study were randomly sub-sampled to match this lower number, minimal network structure was detected and the community could not be discerned (Supplemental Figure 7D). The sample size of the earlier study was therefore likely insufficient to detect the network structure described here.

Both the random bouton and the random claw null models assume that the probability of a PNto-KC connection is independent of its location in the MB main calyx. However, both PN and KC neuronal arbors are known to occupy stereotyped and circumscribed positions within the calyx as a function of cell type (Jefferis et al., 2007; Lin et al., 2007; Tanaka et al., 2004; Zheng et al., 2018). This suggested that cell type-specific neurogeometry might contribute to the observed nonrandom network structure. Therefore a "local random bouton" null model was constructed, in which each $\mathrm{KC}$ claw selects an input at random from the five nearest boutons to it within the MB main calyx (Figure 3A).

The local random bouton model was superior to the prior models, which lacked spatial constraints. In contrast to the random bouton model, it successfully recapitulated the greater number of claws ensheathing community PN boutons (Figure 3B). It also better recapitulated the overconvergence of community PNs onto KCs. In particular, in the observed PN-to-KC network, some KCs received 3-7 claws of input from community PNs, far more than predicted by chance 
(Figure 3C, observed vs. random bouton models). Although the random claw model was constrained to preserve the out-degree of each PN type, it was less successful than the local random bouton model in reproducing the observed distribution of multi-claw convergent inputs from the PN community (Figure 3C, random claw vs. local random bouton models). When individual instances of the local random bouton model were compared to the random bouton model, z-score clustering largely recapitulated the observed PN community (Figure 3D); and zscore clustering following comparison of the observed PN-to-KC network to the local random bouton model failed to reveal the PN community (Figure 3E-F).

The success of the local random bouton model suggested that much of the observed non-random network structure arises from the specific neurogeometry of PNs and KCs. Direct visual examination of the community PN axonal arbors and postsynaptic $\mathrm{KC}$ dendrites bore out this interpretation. Community PN axons were tightly clustered in peripheral regions of the MB main calyx (Figure 4A-B), and the KCs with the most community input showed dendritic arbors localized to four clusters corresponding to these axonal territories (Figure 4C-E). The four clusters of KC dendrites are consistent with four MB neuroblasts (Ito et al., 1997; Lee et al., 1999). Complete reconstruction of an arbitrarily selected bundle of KCs fasciculating tightly in the MB pedunculus (Supplemental Figure 8) also showed regional bias toward the dorsolateral quadrant of the MB main calyx (Figure 4F), where collaterals of the community PNs tended to ramify. Quantification of pairwise inter-bouton distances revealed that community PN boutons were significantly closer to one another than non-community PNs (Figure 4G). Finally, unsupervised hierarchical clustering divided the PN boutons into 4 distinct territories; one of these clusters was made up of nearly all ( 9 of 10) of the community PN subtypes (Figure 4F). Thus the community of super-convergent PN subtypes seems to be generated by neurogeometry, 
as revealed by visual inspection and quantitative analysis of the relevant neuronal arbor structures.

\section{Discussion}

Our results show that the PN-to-KC network in the adult fruit fly has non-random structure. A community of food-responsive PN subtypes converges at above-chance levels onto downstream KCs (Figure 1D). This network structure is set up anatomically: the axons of participating PN subtypes arborize in restricted regions of the MB main calyx, and the dendrites of many postsynaptic KCs are similarly restricted to those regions (Figure 4). The community PN axonal arbor territories s are similar to those obtained in earlier studies based on light microscopy data (c.f. cluster 1 in Figure 4 C\&D Jefferis et al., 2007; Seki et al., 2017; c.f. green cluster in Figure 2 C,E Tanaka et al., 2004). This suggests that the observed PN-to-KC network structure is stereotyped across individuals. The developmental precision required to achieve this structure seems within reach of the fly nervous system, given the highly reproducible geometries of most cell types in the fly brain, including those innervating the MB main calyx (Aso et al., 2014; Lin et al., 2007; Zheng et al., 2018). The PN community we observe in MB is also nearly identical to an independently discovered food-related PN subnetwork formed by axo-axonic synapses between PNs in the lateral horn (c.f. Figure 3F in Bates et al., 2020), suggesting that clustered connectivity of this subset of food-responsive PN types is conserved between brain areas subserving innate (lateral horn) and learned (MB) behavior in the fly.

Why was this structured network connectivity not been seen previously? The likeliest answer may be that past efforts lacked sufficient statistical power to detect the PN community. 
Subsampling of current dataset to match the number of samples of the most thorough of previous efforts (Caron et al., 2013) renders the community of food-reponsive PNs undetectable (Supplemental Figure 7). Differences in results may also be due to the sampling methods used, but until statistical power is sufficient across all methods, it will be challenging to resolve this question. Furthermore, although our effort is the largest to date, additional network structure may be detected if and when the PN-to-KC network is mapped to completion ipsilaterally and contralaterally in the FAFB dataset. Forthcoming additional brain-spanning EM volumes of the adult fly will also be of interest in this regard (e.g. Scheffer et al., 2020). Alternative analysis methods (e.g. Athreya et al., 2017; Jonas and Kording, 2015; Sporns and Betzel, 2016) might also reveal additional networks structure. It will be of interest to learn whether this community is consistent across individuals, and whether it varies as a function of genetic background, neuronal activity levels, and environmental conditions during development (Kremer et al., 2010; Sugie et al., 2018). Even if the observed network structure is conserved across individuals, it is likely that synaptic output from food-responsive $\mathrm{KCs}$ is variable, given that MBON odorant responses are highly variable across individuals (Hige et al., 2015).

What is the functional role of the observed network structure in MB circuit operation? Simplifying models have shown that random connectivity in the PN-to-KC network increases dimensionality and linear separability of neural representation (Litwin-Kumar et al., 2017; Stevens, 2015), indicating that randomly connected Marr motifs may support optimal stimulus classification. However, this assumes that all PNs are activated in a statistically identical fashion. A version of this model incorporating the observed over-convergence of food-responsive PNs onto KCs showed increased discrimination performance for PNs responding to food-related odorants, and decreased performance for the other PN types (Figure 5). This tradeoff calls to 
mind the efficient coding hypothesis, which states that neuronal resources are allocated to match the distribution of natural stimuli, such that more frequently encountered stimuli are sampled more densely (Barlow, 2012; Laughlin, 1981). In a normal fly's life, food-related odorant combinations are presumably encountered more frequently than combinations of arbitrary odorants (Mansourian and Stensmyr, 2015). The efficient coding hypothesis predicts that these more frequently encountered combinations of food-related odorants would be sampled more densely than combinations of arbitrary odorants; and indeed, this is what we observe in the Drosophila Marr motif. Conceptually, this may be thought of as a kind of 'associational fovea', in which more frequently encountered, ethologically relevant combinations of stimuli are sampled more densely (Supplemental Figure 9).

Given the complexity of MB dynamics during learning and recall (Felsenberg et al., 2018; Inada et al., 2017; Owald and Waddell, 2015; Perisse et al., 2016), additional functional characterization of the MB during learning and recall will be needed to determine if the above speculation is correct. Recurrent local microcircuitry is abundant in the MB , including KC-KC synapses (Eichler et al., 2017; Leitch and Laurent, 1996; Liu et al., 2016; Schürmann, 1974), PNPN synapses (Bates et al., 2020), KC-to-PN synapses (Zheng et al., 2018), and extensive connectivity with local and extrinsic neurons (Amin et al., 2020; Butcher et al., 2012; Christiansen et al., 2011; Inada et al., 2017; Lin et al., 2014; Liu and Davis, 2009). It is also unknown whether the cell types involved fire exclusively in all-or-none fashion, or whether synaptic release can be evoked locally (Zhang et al., 2019). This question becomes especially pertinent given the near ubiquity of mixed input/output neurites in the fly brain (with the exception of the finest dendritic processes) are nearly ubiquitous in the fly brain (Bates et al., 2020; Meinertzhagen, 2018; Olsen and Wilson, 2008; Takemura et al., 2017; our unpublished 
observations). Downstream of the PN community, different KC subtypes may also play different roles, an aspect not investigated in the present study. Given these complexities, it may be that richer models will be required to fully describe the effect of the observed network structure (Litwin-Kumar and Turaga, 2019).

The present work joins other studies in which unexpected structure is detected in neuronal networks through quantitative comparison of observed connectivity to null models of neurogeometry (e.g. Bopp et al., 2014; Brown and Hestrin, 2009; Egger et al., 2014; Kasthuri et al., 2015; Lee et al., 2016; Mishchenko et al., 2010). Because connectomics data sets offer the exact positions of all synaptic input and output sites on axonal and dendritic arbors, they provide the opportunity to construct unusually well constrained geometric null models. For many classes of neuronal circuit, connectomics data sets may therefore improve the discoverability of network structure compared to alternative methods. This strength among others illustrates how, although connectomics-style wiring diagrams are by themselves clearly insufficient to explain neuronal circuit function (Bargmann and Marder, 2013), they are a useful scaffolding for integrating data across modalities and generating experimentally testable predictions.

\section{Methods}

\section{Neuron tracing}

Neurons were reconstructed from the whole brain EM dataset of an adult fly (Zheng et al., 2018). Skeleton tracing of neuronal arbors and criteria of synapse annotations are conducted as described previously (Zheng et al., 2018) with the CATMAID tracing environment (Schneider-Mizell et al., 2016). To briefly summarize, all the manually traced neurons were reconstructed with an iterative tracing method by at least two tracers, an initial tracer and a 
subsequent proofreader. The initial tracer reconstructed arbors, followed by systematic review by a different proofreader. When either tracer was not confident about the identifications of a neural process or synapses, they cooperatively examined the image data to reach a consensus. All such sites were further reviewed and resolved by an expert tracer. A chemical synapse was identified if it met at least three of the four following features, with the first as an absolute requirement: 1) an active zone with vesicles; 2) presynaptic specializations such as a ribbon or T-bar with or without a platform; 3) synaptic clefts; and 4) postsynaptic membrane specializations such as postsynaptic densities (PSDs).

Our tracing approach is biased to errors of omission rather than comission. This approach has been shown to have minimal impact on network connectivity in the fly larva (SchneiderMizell et al., 2016). In addition, the present study is focused on the connectivity between PNs and $\mathrm{KCs}$ at a distinctive structure called the microglomerulus, which contains a multitude of synapses between a given PN bouton and its postsynaptic KC claws (Butcher et al., 2012; Leiss et al., 2009; Yasuyama et al., 2002). It is therefore unlikely that the loss of any particular synapse during reconstruction qualitatively affected the analysis described here.

As in Zheng et al. (2018), two reconstruction strategies were used: tracing to classification and tracing to completion. In tracing to classification, in general only backbones and not twigs (microtubule-containing, large diameter neurites, and microtubule-free, fine neurites, respecitvely; Schneider-Mizell et al., 2016) are reconstructed. Tracing is halted once the reconstructed neuronal morphology unambiguously recapitulates that observed by LM or previous EM reconstruction studies for a given cell class. In tracing to completion, all of a given neurite is reconstructed, along with all of its input and output synapses, unless ambiguities in the data make tracing impossible. In some cases tracing to completion is done only within a given 
brain compartment; in the present study, for example, manually reconstructed KCs were traced to completion only within the MB main calyx (see below).

\section{Random sampling of KCs}

Kenyon cells were randomly sampled from within MB pedunculus ("Random Draw KCs") on the right side of the brain. The pedunculus is a tract of fasciculated $\mathrm{KC}$ axons projecting from the posterior of the brain, where $\mathrm{KC}$ dendrites ramify in the the $\mathrm{MB}$ calyx, to the lobes of the MB at the anterior of the brain, where synapses are made between KCs, MBONs and DANs (Technau and Heisenberg, 1982; Figure 1A). All neuronal processes in a transverse plane of pedunculus (section \#4186 in the FAFB dataset) were labelled with seed nodes (2740 in total; Supplemental Figure 1). Seed nodes were randomly selected for reconstruction, which proceeded posteriorly (i.e. retrogradely, in the case of KCs) from the seed node plane. In addition to KCs, the anterior paired lateral (APL) neuron (a wide-field inhibitory neuron; Liu and Davis, 2009), and MB-CP1 (an MBON; Tanaka et al., 2008), were known to have neurites in the pedunculus (Zheng et al., 2018). Therefore tracing to classification was done to determine whether the neuron arising from a given seed node was a $\mathrm{KC}$, using the following morphological criteria. Kenyon cell somata are posterior and slightly dorsal to the MB calyx; each $\mathrm{KC}$ makes a handful of dendritic specializations called "claws" within the calyx; and has a single axon projecting anteriorly, with few branches, in the pedunculus (Aso et al., 2014). The APL neuron (one within the $\mathrm{MB}$ on each side of the brain) has numerous, densely branching and fine neurites ramifying throughout the entire MB. The MB-CP1 neuron similarly branches densely in the pedunculus and calyx. Disambiguating between these neuron types was therefore relatively straightforward, and tracing was halted if the neuron arising from a seed node was determined not to be a KC. 
The Random Draw KCs were reconstructed either manually (440 KCs) or by an automatic segmentation-assisted approach $(916 \mathrm{KCs})$, described below. The total sample size of $1,356 \mathrm{KCs}$ was constrained by the time and resources available for the effort; the overall goal was to obtain as large a sample as possible to maximize statistical power.

\section{Manual tracing of KCs}

Each manually reconstructed $\mathrm{KC}$ was retrogradely traced to completion from at least section 4186 of the FAFB dataset to the posterior of the brain (some were traced to a greater extent). This spans the posterior $\sim 1 / 3$ of pedunculus and the entire MB calyx. In previous work (Zheng et al., 2018), the boutons of all PNs in calyx as well as the glomerular subtypes of all PNs were identified. Typically, each dendritic claw received input from a single bouton (Leiss et al., 2009; Yasuyama et al., 2002). To facilitate downstream analysis (see below), "claw border" tags were applied to each $\mathrm{KC}$ at a node between the "arm" and distal fingers of each $\mathrm{KC}$ claw. The "claw border" tags therefore delineated KC claws post-synaptic to distinct PN boutons. Similarly, "bouton border" tags were applied to the PN arbors within MB main calyx. The majority of reconstructed KCs received olfactory inputs from PNs within MB main calyx. There are 3 main $\mathrm{KC}$ classes, $\gamma, \alpha^{\prime} / \beta^{\prime}, \alpha / \beta$, named according to which of the eponymous lobes at the anterior MB the $\mathrm{KC}$ axon projects (Aso et al., 2014; Crittenden et al., 1998; Lee et al., 1999; Tanaka et al., 2008). Two additional, numerically fewer types of $\mathrm{KC}(\alpha / \beta \mathrm{p}$ and $\gamma \mathrm{d})$ receive non-olfactory inputs such as visual, gustatory, and temperature information via dendritic arbors within MB accessory calyces (Yagi et al., 2016). These were excluded from analysis. All Random Draw KCs were traced to classification anteriorly to section 4186; subtype was assigned depending on which $\mathrm{MB}$ lobe the $\mathrm{KC}$ axon ramified within. 


\section{Automated segmentation-assisted tracing of KCs}

During the $\mathrm{KC}$ reconstruction effort, a segmentation of the FAFB dataset became available (Li et al., 2019). A tracing workflow using this segmentation was therefore adopted. Automated segmentation-derived skeleton fragments were manually concatenated, and the entire resulting arbor was proofread as described above. KC claws were only partially reconstructed, sufficient to define which PN bouton was contained and to identify and annotate at least 3 synapses from the bouton to the claw. Control experiments in which one tracing team manually reconstructed KCs to completion and another independently used the automated segmentation to map PN-to-KC connectivity demonstrated the consistency of results between both approaches in quantifying PN bouton/KC claw connection counts (data not shown).

\section{Conditional input analysis}

To determine whether input to KCs from PNs was independent or conditional on PN type, a new method was devised which we termed "conditional input analysis." The result is a matrix for which a given cell indicates whether, given input from the row PN type, a $\mathrm{KC}$ is more or less likely than chance to get input from the column PN type. This approach also allows for detection of asymmetric conditional input (the case where e.g. KCs on average get more input from type $\mathrm{C}$, given input from type A; but less input from type A, given input from type C). Each observed PN bouton-KC claw connection is treated as a single count. The observed number of counts for a given PN type is compared to the distribution of counts generated using a null model. Several null models were used in this study (see below). For each combination of PN types, a z-score is computed (i.e. how many standard deviations from the mean of the null 
416

417

418

419

distribution the observed number of counts is). Unsupervised K-means clustering of the z-score matrix was used to group matrix entries.

A summary of the steps in conditional input analysis follows; source code is available at https://github.com/bocklab/pn_kc.

Projection neuron types are named after the glomerulus ('Glom') in the antennal lobe that PN's dendrites innervate. Consider types Glom A, B, C, and so on. For a given connectivity matrix,

1. Select all KCs having at least one claw receiving input from a bouton of Glom A.

2. The number of inputs to these KCs from Glom B, C, D, and so on are counted. This provides a count of the number of inputs to the $\mathrm{KC}$ cell population from Glom B-D, given input from Glom A.

3. Repeat (1) - (2) for Glom B, C, D, and so on.

4. For each null model (see below), repeat (1)-(3) above on 1,000 in silico randomizations of the observed PN-to-KC network. This generates the null distributions from which a z-score can be generated for observed connectivity for each PN type pair. A matrix of these z-scores is termed a "conditional input matrix".

7. Apply K-means clustering to the conditional input matrix. The K-means algorithm (MacQueen, 1967) clustered PN types into groups with equal variances and the cluster number of each PN type is used to re-order both the columns and rows of the z-score matrix.

K-means clustering of the conditional input matrix groups glomeruli with similar z-scores together, and therefore reveals subsets of PNs that provide more (or less) input than predicted by a given null model. Over-convergence of inputs (red in our figures) is more strongly detected by this approach, since the random bouton null model (see below) can result in PN types having a 
439

440

441

442

small number of boutons to have zero KC outputs. This, in turn, lowers the magnitude of negative z-scores (since the mean of the null model values is already low).

\section{Null models of PN-to-KC connectivity}

Three null models were used: (1) random bouton model, (2) random claw model, and (3) local random bouton model.

In the random bouton model, each Random Draw KC claw is reassigned, with replacement, to a randomly selected PN bouton in the calyx. On average, therefore, the number of outputs provided by PN type (i.e. out-degree per PN type) will be proportional to the number of boutons that belong to that type. The number of claws for each KC (i.e. in-degree per KC) is also maintained. To apply conditional input analysis to the data of Caron et al. (2013) using this null model, the bouton counts per PN type obtained from the present work were used (Supplemental Figure 7), since bouton counts per PN type were not generated in that study.

In the random claw model, each PN bouton is reassigned claws at random, without replacement. The number of claws so assigned is equal to the number of claws ensheathing that bouton in the observed PN-to-KC network. Thus in this randomization, the number of claws receiving input from a given PN type (i.e. out-degree per PN type) and the number of claws each $\mathrm{KC}$ has (i.e. in-degree per KC) are maintained.

In the local random bouton model, each claw of each $\mathrm{KC}$ is randomly assigned to one of its five nearest boutons (including the one it ensheathed in the observed network), with replacement. Distances were measured between claw and bouton centroids. In this randomization, $\mathrm{KC}$ in-degree and geometric constraints on connectivity are preserved. 


\section{Covariance analysis of connectivity}

Covariance analysis (Newman, 2018) is a commonly used measure of whether two inputs occur more frequently than predicted by chance and as such is an alternative to the conditional input analysis described above. Its output is a matrix of p-values of input rates compared to the expected distribution arising from given null model. The procedure is summarized as follows.

1. The covariance measure for each pair-wise combination of PN types was computed for the observed connectivity.

2. The observed PN-to-KC connectivity was randomized 1,000 times using the random bouton model. For each randomization, a covariance matrix of PN types was computed.

3. For each pairwise combination of PN types, a $\mathrm{p}$ value is estimated by counting how often the randomized covariance was great than or equal to the observed covariance. A p value of less than 0.05 (significance level) implies the probability of obtaining such a covariance in a random network is low, and the alternative hypothesis of seeing such an observed value in a null model is therefore rejected. The results are shown in a p-value matrix (Supplemental figure $4 \mathrm{~A}$ D) in which each cell represents a p value for a given pair of glomeruli indicated in the corresponding row and column labels.

4. The p-value matrix was re-ordered either using the Fig 1D clustering order (Supplemental Figure 4 A, C) or using order given by K-means clustering (Supplemental Figure 4 B, D). To cluster statistically significant but numerically small $p$ values, $K$-means clustering was performed on a binary version of the $p$-value matrix wherein all $p$ values less than 0.05 were set to 1 , and otherwise to 0 .

For the analysis of synaptic connectivity (Supplemental Figure 4C, D), covariance measures were directly calculated from synapse counts, using only the manually reconstructed 
Random Draw KCs (whose dendritic arbors in MB calyx were reconstructed to completion; see Manual Tracing of KCs, above). To generate the null model of synaptic connectivity, the boutonclaw binary network is randomized and each bouton-claw connection is assigned a synapse count that was randomly drawn (with replacement) from the distribution of number of synapses per claw.

\section{Clustering analysis of PN boutons}

Each PN type was classified and each the bouton in MB calyx was annotated in previous work (Zheng et al., 2018). Using these annotations, skeleton reconstructions of each bouton were extracted. Pairwise NBLAST scores on the bouton skeletons were computed (Costa et al., 2016) and clustered by Ward's algorithm (Murtagh and Legendre, 2014). NBLAST is a similarity measure for both shape and position; in this case, because the skeletons within each bouton were small, clustering is likely based mostly on bouton position. The probability of bouton arbors being in a given location in calyx was estimated following the approach of Bates et al. (2020). In brief, the bouton skeletons were resampled evenly at $0.1 \mu \mathrm{m}$ intervals. A Gaussian kernel density estimate (KDE) was used to fit the number of skeleton nodes per unit space (cubic $\mu \mathrm{m}^{-1}$ ) for each of the two projected dimensions ( $\mathrm{x}, \mathrm{y}$ or $\mathrm{x}, \mathrm{z})$. The density map therefore reflects the probability (point density function, PDF) for boutons of a given PN type to be found at a given location in MB calyx. The PDF is normalized to the same scale $\left(0-2.5 \times 10^{-9}\right)$ for each of the four groups. The boundaries of MB calyx were generated from an nc82 (synapse)-stained template brain aligned to the FAFB image volume as described in Zheng et al. (2018).

\section{Comparison of postsynaptic KC counts between FAFB and hemibrain datasets}


During preparation of this manuscript, a segmentation of a portion of a second adult fly brain became available in preprint form (the 'hemibrain'; Scheffer et al., 2020). In the hemibrain dataset, all PNs and $\sim 2,000 \mathrm{KCs}$ on the right side of the brain were segmented as part of a largescale proofreading effort (50 person-years over $\sim 2$ calendar years). As of this writing, the publicly available hemibrain segmentation does not demarcate PN bouton and KC claw boundaries, preventing straightforward application of our analysis approach. We used the hemibrainr package (https://github.com/flyconnectome/hemibrainr) to download the connectivity matrix between all PNs and KCs from the dataset server (hemibrain v. 1.0.1, https://neuprint.janelia.org). The connectivity matrix is then binarized such that each unique pair of $\mathrm{PN}$ and $\mathrm{KC}$ with 3 or more synapses is defined as one connection and otherwise zero. For a $\mathrm{PN}$, the number of connections is equivalent to the number of KCs postsynaptic to the PN. Connections for different PNs of a common type are summed and divided by the total number of connections in the entire binary connectivity matrix. The percentage of connections for each PN type is used to compare to the same number from FAFB (Supplemental Figure 2B-C).

\section{Modeling}

The PN-to-KC network model was a modification of earlier models used in the larval and adult fly (Eichler et al., 2017; Litwin-Kumar et al., 2017, respectively). In these models, simulated activities across all PNs are created for each stimulus odor. Each stimulus is randomly associated with one of two categories with equal probability. The PN activity (signal) is generated by drawing independently from a rectified unit Gaussian distribution corrupted by Gaussian noise (s.d. 0.2). To probe the effect of the observed overconvergence of community PN types (10 types comprising 16 individual PNs), for each stimulus, 16 modeled PNs were activated 
531

532

(i.e. Gaussian activity patterns were created). Within the 16 activated PNs, the fraction of community PNs was varied as follows: 0 (16 non-community PNs), 0.125 (2 community PNs, 14 non-community PNs), 0.25 (4 community PNs, 12 non-community PNs), 0.375 (6 community PNs, 10 non-community PNs), 0.5 (8 community PNs, 8 non-community PNs), 0.625 (10 community PNs, 6 non-community PNs), 0.75 (12 community PNs, 4 non-community PNs), 0.875 (14 community PNs, 2 non-community PNs), 1 (all 16 community PNs). These fractional values comprise the $\mathrm{x}$-axis of Figure 1F. For fractional values less than 1, activated PNs were randomly selected from the 16 community PNs. For all fractional values, non-community PNs were randomly selected from the population of 97 non-community PNs. Gaussian noise with standard deviation 0.2 was then added to the activity levels of all PNs. Kenyon cell activity is given by multiplying PN activity by the matrix of PN-to-KC connections $m=\Theta(h-\theta)$, where $\theta$ is a threshold whose values are picked for each $\mathrm{KC}$ such that each $\mathrm{KC}$ is active with a probably of $f(\mathrm{f}=0.05$, also called coding level $)$ for each stimulus. The $\Theta$ is a rectification term. The $h$ represents input activity provided by each PNs multiplied by their corresponding number of synapses to the $\mathrm{KC}$. $\mathrm{KC}$ activity patterns were used to train a maximum-margin classifier, and the goal is to predict the pre-assigned one of two categories. In the testing phase, the same set of PN activity patterns corrupted with different applications of Gaussian noise were used as input, and the resulting $\mathrm{KC}$ activity patterns were given to the trained classifier to predict which of the two categories each stimulus belongs to. Error rates of the prediction from 1,000 simulations were used to evaluate classifier performance. Because this is a two-alternative classification task, the expected error rate for chance performance is $50 \%$. For reporting error rate results (Figure $1 \mathrm{~F}$, Supplemental Figure 5A-B), standard errors of the mean (s.e.) are used as the goal is to compare mean error rate of different models. 
The model requires synapse counts between each connected PN-KC cell pair. In Figure $1 \mathrm{~F}$ and Supplemental Figure 5A, the synaptic counts between PNs and manually reconstructed Random Draw KCs were used. In Supplemental Figure 5A, the same model is implemented except that 38 food PNs (Supplemental Table 1) are chosen to be activated. In the activated foodPN datum (red dot), 38 food PNs are activated with simulated Gaussian activity patterns and all PNs, including the food PNs, are corrupted with Gaussian noises (s.d. 0.2). In the null model distribution (blue histogram in Supplemental Figure 5A), for each simulation (one count in the histogram), a random set of $38 \mathrm{PNs}$ are picked to be activated and error rates of the classifier are computed to evaluate performance of the models. In Supplemental Figure 5B, each KC's connections to a given PN were randomly reassigned (with replacement) to different PN, and 16 PNs, with varying proportion of community PNs, are activated using the same model implementation as in Figure 1F.

\section{Statistics}

When comparing two or more distributions, if the data are categorical (e.g. Figure 2A, 3C, Supplemental Figure 2C) a Chi-square test is used. When the data are continuous (e.g. Figure 4G, Supplemental Figure 3B-D, 6B), a Kolmogorov-Smirnov test (K-S test) is used. When a distribution is compared with a observed datum (i.e. a single data point), as in each cell of the conditional input matrices (e.g. Figure 1D, 2D, 3D-F, Supplemental Figure 3E, 6, 7A, CD), in Figure 2B, C, 3B, and in Supplemental Figure 5A, a z-score (see Conditional input analysis, above) is computed. 
${ }_{576}$ Figure legends

577

578

579

580

581

582

583

584

585

586

587

Figure 1

(A) Schematic of olfactory pathway. Odorants bind to olfactory receptor neurons (ORNs) in the fly antennae and activate a stereotyped subset of glomeruli in the antennal lobe (AL). ORNs in a specific glomerulus provide olfactory inputs to a given class of PNs and the PNs can be classified into $\sim 51$ types based on their originating antennal lobe glomeruli. In each hemisphere of a fly brain, $\sim 150$ PNs project to two higher brain regions, MB and lateral horn (LH). In the calyx of the MB, the PNs synapse onto $\sim 2,200 \mathrm{KCs}$. The KCs then converge onto a small number of mushroom body output neurons (MBONS, $\sim 34)$ at the medial and vertical lobes of the MB. Modification of synapses between KCs and MBONs likely underlies olfactory learning and memory in the fly (Barnstedt et al., 2016; Guven-Ozkan and Davis, 2014; Heisenberg, 2003).

(B) Electron microscopy reconstruction of the dendrites of a $\mathrm{KC}$ and its olfactory input from PNs in the MB calyx. Kenyon cell dendrites terminate as claw-like elaborations; each claw receives a variable number of synapses (numbers in white) from a single ensheathed PN bouton. Each KC gives rise to a small number of claws (mean \pm s.d., $5.2 \pm 1.6$; Supplemental figure 1).

(C) Schematic of conditional input analysis of the PN-to-KC network. Each PN-to-KC connection is treated as binary: if a claw receives three or more synapses from a PN bouton, the $\mathrm{KC}$ is considered as receiving one input from that $\mathrm{PN}$ type; otherwise it is treated as zero. Input counts across all $\mathrm{KCs}$ are then compared to a randomized null model. In the example, given input from $\mathrm{PN}$ type 'A', KCs are more likely to receive input from $\mathrm{PN}$ type ' $\mathrm{C}$ ' and less likely to receive input from PN type 'B'. A matrix is used to represent the population of these conditional input probabilities. Each row in the matrix represents the probability that, given input from the PN 
type for that row, KCs are more (red) or less (blue) likely to get input from the PN types in the columns. Re-ordering of the matrix by K-means clustering helps illustrate these relationships. The color scale for each cell in the matrix indicates the z-score, i.e. the number of standard deviations (s.d.) between the observed number of inputs and the mean number of inputs arising from the null model.

(D) Structured PN-to-KC connectivity against the random bouton null model. Conditional input analysis was applied to 1,356 randomly sampled $\mathrm{KCs}$ on the right side of the fly brain. A specific group of PNs ('community' PNs, type names in bold) were found to provide above-chance levels of convergent input to downstream KCs. Olfactory PN types are color-coded according to the category of odorants to which they respond. All community PNs have been reported to primarily encode food-related odors (Supplemental Table 1).

(E) Schematic of PN-to-KC network model. In the PN input layer, an olfactory response is represented as a signaling response within a subset of PNs, mixed with Gaussian noise across all PNs. Each of these activity patterns is then assigned a positive or negative valence. Kenyon cell activity is the product of the observed PN-to-KC connectivity matrix and simulated PN activity, subject to a sparseness constraint such that only $5 \%$ of KCs are active at any time. The classifier learns to predict the pre-assigned valence based on a readout of $\mathrm{KC}$ activity. Performance is quantified by calculating the error rates of the classifier prediction.

(F) Using the observed PN-to-KC connectivity, discrimination of inputs from community PNs is superior to that of inputs from non-community PNs. Error bars are standard errors (s.e.) of the mean across 1,000 simulations. For each simulation, a randomly selected subset of PNs are activated. A larger number of activated community PNs leads to better discrimination performance. 
622

623

624

625

626

627

628

629

630

631

632

633

634

635

636

637

638

639

640

641

Figure 2

(A) Biased sampling of PN inputs by KCs. Each bar in the x-axis represents a PN and the y-axis shows the number of claws that receive input from each PN in the random bouton null model (blue) and in the observed network (orange). PNs are grouped by type (i.e. glomerular class) and colored by behavioral significance (as in Figure 1D). Community PN types are underlined. In the observed network, community PNs are usually presynaptic to more KCs than predicted by the random bouton null model (error bars, s.d. of 1,000 random networks; Chi-square test $\mathrm{p}<$ $\left.1 \times 10^{-10}\right)$.

(B) Kenyon cells over-sample inputs from community PNs. The observed number of claws receiving input from community PNs (red dot) was greater than the mean of the random bouton null model (distribution of 1,000 random networks; random bouton null model, mean \pm s.d., $1412.5 \pm 34.0$; observed, 1901; z-score, 14.3).

(C) Community PNs provide convergent input onto postsynaptic KCs. The observed number of KCs receiving one or more inputs from community PNs (red dot) was lower than the mean of the random bouton null model (distribution from 1,000 random networks; random bouton null model: mean \pm s.d., $903.4 \pm 17.3$; observed, 844; z-score, 3.4).

(D) Overconvergent PN-to-KC connectivity contributes to the PN community. Conditional input analysis was applied to the observed PN-to-KC connectivity using the random claw null model, in which PN-to-KC connections are randomized while holding constant both the number of $\mathrm{KC}$ claws postsynaptic to each PN type and the number of input PN boutons to each KC. The PN 
642

643

644

645

646

647

648

649

650

651

652

653

654

655

656

657

658

659

660

661

662

663

community still emerges, despite the fact that the random claw model incorporates the greaterthan-chance output from community PN types (Figure 2A).

Figure 3

(A) Schematic for the local random bouton null model. Each claw of each $\mathrm{KC}$ is randomly assigned to one of the five nearest PN boutons. In observed PN-to-KC network (upper), one claw from the $\mathrm{KC}$ receives input from a PN bouton (purple). The claw is randomly assigned to a different neighboring PN bouton (green).

(B) The local random bouton model recapitulates the greater output of community PNs. The observed number of claws receiving inputs from community PNs (red dot) was compared to the number of claws with community inputs in the random bouton model (green histogram) and the local random bouton model (blue histogram). Each distribution represents 1,000 random networks from the null models. Observed (1901) vs. random bouton model (mean \pm s.d., 1412.5 \pm 34.0 ), z-scores 14.3. N.b. the random claw null model is constrained to have the observed number of claws postsynaptic to community PNs, and therefore is not considered here.

(C) Individual KCs have multiple claws postsynaptic to community PNs boutons. Distributions are shown for the observed network, as well as the means of the random bouton, random claw, and local random bouton null models (error bars, \pm s.d.; observed vs. random bouton null model, Chi-square test $\mathrm{p}<1 \times 10^{-10}$; observed vs. random claw null model, Chi-square test $\mathrm{p}<1 \times 10^{-10}$; observed vs. local random bouton null model, Chi-square test $\mathrm{p}<0.028$ ).

(D) The local random bouton model recapitulates some of the PN community. Conditional input analysis was applied to a representative instance of the local random bouton model, with the 
664

665

666

667

668

669

670

671

672

673

674

675

676

677

678

679

680

681

682

683

684

685

random bouton model as the null model. The local random bouton model captures predominantly the same cluster of community PNs (except one PN class, DM5). Community PN types are in bold, and all PN types are color-coded by their response categories as in Figure 1D.

(E) Conditional input analysis of the observed connectivity using the local random bouton model as the null model shows no connectivity structure contributed by the community PNs. The remaining network structure is due to biased sampling of other PN types (see Figure 2A).

(F) The same matrix shown in (E) but with columns and rows ordered as in Figure 1D. The cluster of community PNs, as seen in Figure 1D, is not seen here, as the community PN network structure is largely recapitulated by the local random bouton null model.

Figure 4

(A) Reconstructed PNs project from AL to two higher brain centers, MB and LH. Community PNs (green) have regionalized projection patterns in MB and LH compared to non-community PNs (white/purple).

(B) Frontal view of MB calyx showing reconstructed PN axon arbors; colors as in (A).

(C) Same as (B), with the addition of the 6 manually reconstructed KCs receiving 6 or more bouton inputs from community PNs. The dendritic arbors of these KCs (red) overlap with the community PN axon territories (green).

(D) Posterior view of MB calyx showing 46 reconstructed KCs that receive 5 or more inputs from community PNs. The dendrites and soma of the KCs, respectively, are segregated into 4 clusters (assigned 4 arbitrary colors) that may correspond to the 4 different neuroblasts of KCs in development (Ito et al., 1997; Lee et al., 1999). 
686

687

688

689

690

691

692

693

694

695

696

697

698

699

700

701

702

703

704

705

706

(E) Dorsal view of calyx shows 4 different clusters of the same set of KCs as shown and colorized in (D). The cluster axonal bundles also fasciculate in the pedunculus (bottom of figure).

(F) Frontal view of calyx shows PN collaterals (colors as in A) and reconstructions of all KCs from a single bundle ("bundle KCs"). The bundle KC dendrites ramify in the dorsal-lateral quarter of the calyx, overlapping extensively with the community PN axonal arbor territory.

(G) Community PN boutons are closer to each other than non-community PN boutons. Each count represents the distance between a bouton and its nearest same-type bouton (blue: community PN bouton pairs; green: non-community PN bouton pairs; K-S test $\mathrm{p}<1 \times 10^{-10}$ ) (H) Unsupervised clustering reveals community PN boutons are spatially colocated in the MB calyx. (upper) Hierarchical clustering based on bouton arbor NBLAST score divides the PNs into four different groups. Nine out of ten community PNs belong to the same group. The y axis of the dendrogram represents Euclidean distances and is cut at 1.5 to divide different PN subtypes into 4 clusters. (lower) Bouton density maps of the four different groups. Colors correspond to the four groups shown in the dendrogram. Color intensity represents density of bouton arbors in a unit space (cubic $\mu \mathrm{m}^{-1}$ ) and is based on normalized point density function (PDF, Methods).

\section{Supplemental Figure 1}

(A) Schematic of MB anatomy, as in Figure 1A. Kenyon cell axons fasciculate and project anteriorly in parallel within the pedunculus. The blue line in the pedunculus indicates the location of the transverse plane where KCs were randomly sampled for reconstruction (B - C). 
707

708

709

710

711

712

713

714

715

716

717

718

719

720

721

722

723

724

725

726

727

(B) Subarea of a frontal section from the whole-brain EM volume, showing the cross-section through pedunculus (blue false color) used for random sampling (C).

(C) Randomly sampled KCs in the pedunculus. The cross-section of each randomly sampled KC axon is annotated with a magenta dot. All neurite cross-sections within the pedunculus were initially annotated (not shown); if a neurite was randomly sampled for reconstruction that turned out not to be a KC, it was discarded from further analysis. N.b. a discrete region in the middle of the pedunculus is occupied by other cell classes such as APL and non-olfactory KCs from accessory calyces (i.e. KC- $\alpha / \beta p$ and $\mathrm{KC}-\gamma \mathrm{d}$ ), hence there are no magenta points in this region.

\section{Supplemental Figure 2}

(A) Distribution of number of claws per KC for all randomly sampled KCs (mean \pm s.d., $5.2 \pm$ 1.6).

(B) The number of postsynaptic KCs per PN type is consistent between the current study and the connectome deriving from the recent 'Hemibrain' dataset (v. 1.0.1; Scheffer et al., 2020). Each point represents a PN type; three or more synapses between a unique $\mathrm{PN}-\mathrm{KC}$ pair is counted as an individual PN-KC connection. Since the two datasets have different numbers of reconstructed $\mathrm{KCs}$, output from each PN type is represented as a percentage. There is a tight correlation across the two datasets $\left(r^{2=} 0.83\right.$; blue-gray, $95 \%$ confidence interval along the regression line).

(C) The same data as in (B), with PN types identified.

Supplemental Figure 3 
728

729

730

731

732

733

734

735

736

737

738

739

740

741

742

743

744

745

746

747

748

749

(A) Figure 1D, with colored boundaries delineating the matrix subregions for which z-score distributions are shown in (B-D). The distributions are significantly different (B, green; C, yellow; D, blue).

(B) Distribution of z-scores for community PN types (green area in panel A).

(C) Distribution of z-scores for PN types weakly clustering with the community PNs (yellow area in panel A). It is significantly different from the community PN distribution (K-S test $\mathrm{p}<$ $\left.1 \times 10^{-10}\right)$.

(D) Distribution of z-scores for remaining PN types (blue area in panel A). It is significantly different from the community PN distribution (B, above; K-S test $\mathrm{p}<1 \times 10^{-10}$ ) and the weakly clustering PN types (C, above; K-S test $\mathrm{p}<1 \times 10^{-10}$ ).

(E) Conditional input analysis of a single representative network from the random bouton model, shows no clustered structure in the z-score matrix. The random bouton model was also used as the null model. Any connectivity structure that deviates from the null model will manifest as clusters of high or low z-scores ( 2 s.d. or more as compared to the mean of the null model) in the matrix. No discernible cluster is seen after re-clustering of the z-score matrix, showing that the observed clustering is unlikely to be an artifactual result from an expected distribution of random values.

Supplemental Figure 4

(A) Co-variance analysis (Methods) of the observed PN-to-KC connectivity. This approach generates a symmetric matrix of p-values for PN type combinations. The lower the p-value, the 
less likely the observed convergence of the PN type pair onto postsynaptic KCs is expected to occur by chance. Values $<0.05$ are color coded in yellow; others are black. The column-row ordering of PN types is the same as in Figure 1D. The PN community is discernible as a mostly yellow square at the top left. PN type response categories are color coded as in Figure 1D. (B) As in (A), except the covariance matrix is reordered using unsupervised K-means clustering on p-values. The PN community (type names in bold) is reconstituted following this reclustering. Two weaker clusters of PN types (red and blue squares, overlaid) are discernible. (C) As in (A), except covariance between synapse counts between each PN and KC pair was quantified. The PN community is still discernible.

(D) As in (B), except covariance between synapse counts between each PN and KC pair was quantified. As with (A-C), the PN community types comprise the dominant cluster, confirming that the main finding is robust to different analysis methods.

(E) The z-score matrix from the conditional input analysis (Figure 1D) is re-ordered with the order given by K-means clustering of the co-variance matrix as shown in (B). The re-ordering reveals the same two weaker clusters of PNs as seen in (B).

(F) Anterior view of MB calyx shows axon collaterals of reconstructed PNs of the types shown in the top left weak cluster in (B), demarcated by a red square. The PN collaterals occupy a conscribed territory within the MB main calyx.

(G) As in (F), except the PN types are from the second weak cluster in (B), demarcated by a blue square. 
(A) In a model using the observed PN-to-KC network, stimulus discrimination by food odorantresponsive PNs is superior to discrimination by other PN types. The classifier seeks to discriminate different sets of PN activity patterns based on the $\mathrm{KC}$ responses, which are integrated via the observed PN-to-KC connectivity (for schematic see Figure 1D). In each set of PN activity patterns, 38 PNs are activated. The red $\operatorname{dot}(x=0.03)$ indicates average performance from 1,000 simulations of the model with activation of all food-responsive PNs (38 in total, including PN types that are not part of the community identified in Figure 1D). The distribution (mean 0.057, s.e. 0.007 ) shows error rates from 1,000 sets of 38 non-food PNs that are activated. Each data point represents the average error rate for a set of 38 PNs randomly selected from all non-food PNs. The number of non-food PNs that are activated (38) is kept consistent with the total number of food PNs. Observed vs. blue histogram, $\mathrm{z}$-score $-4.0, \mathrm{p}<1 \times 10^{-4}$.

(B) With a randomized PN-to-KC network, activating varying fractions of community PNs results in unchanging classification performance. Each $\mathrm{KC}$ claw is randomly assigned to a PN with equal probability for each PN, and each PN-claw pair is assigned a number of synapses randomly drawn from the distribution of synaptic counts for all manually reconstructed claws. For each plotted data point, the same number of PNs (16) is activated, but with a varying fractions of community PNs (indicated in $\mathrm{x}$-axis). Each data point is the average of 1,000 simulations, each of which represents a combination of randomly selected community PNs and non-community PNs according to the indicated fractions (e.g. in the case of $0.5,8$ randomly selected community PNs and 8 randomly selected non-community PNs).

\section{Supplemental Figure 6}


(A) Conditional input analysis was applied to a representative instance of the local random bouton model, with the random bouton model as the null model.Conditional input analysis was applied to a representative instance of the random claw model, with the random claw model as the null model. No cluster of high or low z-scores ( 2 s.d. or more as compared to the mean of the null model) is seen after K-means re-clustering of the z-score matrix.

(B) Distribution of z-scores from conditional input analysis using random bouton null model (zscores in Figure 1D; i.e. observed vs. random bouton model, mean -0.044, s.d. 2.11) and analysis using the random claw null model (z-scores in Figure 2D; i.e. observed vs. random claw model, mean -0.058 , s.d. 1.47). Blue vs. orange distributions, K-S test $\mathrm{p}<1 \times 10^{-10}$. Variance of z-scores is lower using the random claw model than the random bouton model, indicating that the random claw model better captures the observed network structure.

\section{Supplemental Figure 7}

(A) Conditional input analysis of PN-to-KC connectivity data from Caron et al. (2013), using the random bouton null model (Methods). A weak cluster of overconvergent PN types is seen in the lower right corner of the matrix, consistent with the previously reported set of types making the most output onto KCs (Supplemental Figure 1, Caron et al., 2013). This cluster does not overlap strongly with the overconvergent PN community described in the present work (PN type names color coded and bolded as in Figure 1D).

(B) Histogram view of data underlying (A). The y-axis shows the mean number of claws receiving input from each PN type in the random bouton null model (blue) and in observed counts the Caron et al. (2013) data (yellow). PN types are ordered as in (A). 
816

817

818

819

820

821

822

823

824

825

826

827

828

829

830

831

832

833

834

835

836

837

(C) Conditional input analysis as in (A), except using the random claw null model, which incorporates the observed output rate of each PN type. No overconvergent PN type clusters are discernible using this model.

(D) Conditional input analysis of a representatively randomly sampled subset of PN-to-KC connectivity from the current study shows only weak clustering (most z-scores $<2$ ). The number of KCs, and KCs per claw, was held equal to that of the Caron et al. (2013) study.

\section{Supplemental Figure 8}

(A) Fasciculating KCs ('bundle' KCs) in the pedunculus. A transverse plane image of the pedunculus (shaded blue) shows a discrete bundle of KCs (blue outline) that was completely reconstructed. The black rectangle delineates the subarea shown in (B).

(B) Magnified view of the cross-sectional profile of bundle $\mathrm{KC}$ axons (magenta dots) in the pedunculus.

\section{Supplemental Figure 9}

(A) A high dimensional olfactory space is represented schematically here as two-dimensional (x,y axes). Kenyon cells (red dots, upper panel) may be considered as points in this space, with positions defined by their PN inputs. In a random PN-to-KC wiring, the probability that a $\mathrm{KC}$ is responsive at a particular position along the y dimension is independent from its responses along the $\mathrm{x}$ dimension (lower panel).

(B) In the PN-to-KC network structure we observe, $\mathrm{KCs}$ receive convergent input from PNs responsive to food-related odorants more often than predicted by chance. Schematically, this 
may be represented as a non-uniform distribution of $\mathrm{KCs}$ within the high dimensional olfactory space defined by PN inputs, analogous to the denser sampling of a visual scene in the fovea of the retina. In this case, the probability that a given $\mathrm{KC}$ is responsive to a particular odor along the $\mathrm{y}$ axis is not independent from whether it is responsive to an odor on the $\mathrm{x}$ axis (lower panel). Assuming the fly has a constant number of KCs regardless of network structure (i.e. the number of red dots is the same no matter what), then within the 'associational fovea' (green circle, upper panel), the probability is substantially increased, and everywhere else the probability is slightly decreased (lower panel).

Supplemental Table 1

\begin{tabular}{|l|l|l|}
\hline $\begin{array}{l}\text { PN } \\
\text { types }\end{array}$ & $\begin{array}{l}\text { Behavioral } \\
\text { Significance }\end{array}$ & Literature \\
\hline D & Unknown* & $\begin{array}{l}\text { *multiple: aversive (Knaden et al., 2012), pheromonal (Lebreton et al., } \\
\text { 2017), and yeast volatiles (ethyl 3-hydroxyhexanoate, Tsakiris et al., 2010) }\end{array}$ \\
\hline DA1 & Pheromonal & (Kurtovic et al., 2007) \\
\hline DA2 & Aversive & (Stensmyr et al., 2012) \\
\hline DA3 & Unknown & \\
\hline DA4l & Aversive & (Badel et al., 2016) \\
\hline DA4m & Unknown & \\
\hline DC1 & Egg-laying & (Dweck et al., 2013) \\
\hline DC2 & Aversive & (Knaden et al., 2012) \\
\hline DC3 & Food & (Ronderos et al., 2014) \\
\hline DC4 & Aversive & (Ai et al., 2010) \\
\hline DL1 & Unknown & \\
\hline DL2d & Food & (Mansourian and Stensmyr, 2015) \\
\hline DL2v & Food & (Mansourian and Stensmyr, 2015) \\
\hline DL3 & Pheromonal & (van der Goes van Naters and Carlson, 2007) \\
\hline DL4 & Aversive & (Ebrahim et al., 2015) \\
\hline DL5 & Aversive & (Knaden et al., 2012) \\
\hline DM1 & Food & (Semmelhack and Wang, 2009) \\
\hline DM2 & Food & (Schubert et al., 2014) \\
\hline DM3 & Food & (Semmelhack and Wang, 2009) \\
\hline DM4 & Food & (Badel et al., 2016; Semmelhack and Wang, 2009) \\
\hline DM5 & Aversive & (Semmelhack and Wang, 2009) \\
\hline DM6 & Food & (Schubert et al., 2014) \\
\hline DP1I & Food & (Mansourian and Stensmyr, 2015; Silbering et al., 2011) \\
\hline DP1m & Food & (Semmelhack and Wang, 2009) \\
\hline V & Aversive & (Suh et al., 2004) \\
\hline VA1d & Pheromonal & (van der Goes van Naters and Carlson, 2007) \\
\hline & &
\end{tabular}




\begin{tabular}{|l|l|l|} 
VA1v & Pheromonal & (Dweck et al., 2015b) \\
\hline VA2 & Food & (Semmelhack and Wang, 2009) \\
\hline VA3 & Unknown & \\
\hline VA4 & Food & (Laissue and Vosshall, 2008) \\
\hline VA5 & Unknown & \\
\hline VA6 & Food & (Mansourian and Stensmyr, 2015; Schlief and Wilson, 2007) \\
\hline VA7I & Aversive & (Mansourian and Stensmyr, 2015) \\
\hline VA7m & Unknown & \\
\hline VC1 & Unknown & \\
\hline VC2 & UnknownT & $\begin{array}{l}\text { I unclear: dietary antioxidants (Dweck et al., 2015a); suppress oviposition, } \\
\text { (Chin et al., 2018) }\end{array}$ \\
\hline VC3I & Food & (Laissue and Vosshall, 2008) \\
\hline VC3m & Food & (Laissue and Vosshall, 2008) \\
\hline VC4 & Unknown & \\
\hline VC5 & Egg-laying & (Hussain et al., 2016) \\
\hline VL1 & Unknown & \\
\hline VL2a & Food & (Grosjean et al., 2011) \\
\hline VL2p & Aversive & (Hamada et al., 2008) \\
\hline VM1 & Food & (Min et al., 2013) \\
\hline VM2 & Food & (Root et al., 2007) \\
\hline VM3 & Food & (Mansourian and Stensmyr, 2015) \\
\hline VM4 & Unknown & \\
\hline VM5d & Food & (Hallem and Carlson, 2006) \\
\hline VM5v & Food & (Mansourian and Stensmyr, 2015) \\
\hline VM7d & Food & (Laissue and Vosshall, 2008) \\
\hline VM7v & Unknown & \\
\hline VP1 & Others & temperature (Enjin et al., 2016) \\
\hline VP2 & Others & temperature (Frank et al., 2015; Liu et al., 2015) \\
\hline VP3 & Others & temperature (Enjin et al., 2016) \\
\hline
\end{tabular}

\section{Acknowledgements}

We thank: Greg Jefferis and Paavo Huoviala for substantial contributions to the literature search to classify PN types for behavioral significance; Greg Jefferis, Eyal Gruntman, Shaul Druckman, Larry Abbott, Ashok Litwin-Kumar, and Marcus Meister for helpful discussion of preliminary data; Jacob Ratliff, Shahrozia Imtiaz, Benjamin Gorko, Arynne Boyes, Adam John, Emily Moore, Ben Koppenhaver, Philipp Ranft, bailey harrison, Sri Murthy, Ala Haddad, Addy Adesina, Ashley Scott, Chelsea Marlin, Emily Wissell, Zachary Gillis, Saba Ali, Gabrielle Allred, Spencer Waters, Lisa Marin, Annie Scott, Sarah Mohr, Michael Lingelbach, Emma Spillman, Aidan Smith, Teri Ngo, Jordan Dunlap, Bindu Gampah, Melissa Ryan, Nethan Reddy, Adam Fischel, Markus Pleijzier, Arlo Sheridan, Kabas Abou Jahjah, Amelia Edmondson-Stait, Ilenia Salaris, Ruchi Parekh, Austin Warner, Winston Chen, Ruairi Roberts, Julia Gonzales, Laurin Bueld, Cory Ardekani, Razi Rais, Niles Ribeiro, Teresa Neves for Kenyon cell reconstructions; Noah Nelson, for pilot software for analysis of the PN-to-KC connectivity graph 
at the level of boutons and claws; J. Scott Lauritzen, for help coordinating reconstruction efforts. Funding: Howard Hughes Medical Institute; Wellcome Trust collaborative award 203261/Z/16/Z; NIMH BRAIN Initiative award 1RF1MH120679-01.

\section{Availability of Source Code and Neuronal Reconstructions}

The neuronal reconstructions and source code underlying the analyses presented here are available at: https://github.com/bocklab/pn_kc. Neuronal reconstructions are also available at the Virtual Fly Brain Project

(https://v2.virtualflybrain.org/org.geppetto.frontend/geppetto?id=vfb_site/overview.htm).

Ai, M., Min, S., Grosjean, Y., Leblanc, C., Bell, R., Benton, R., and Suh, G.S. (2010). Acid sensing by the Drosophila olfactory system. Nature 468, 691-695.

Albus, J.S. (1971). A theory of cerebellar function. Mathematical Biosciences 10, 25-61.

Amin, H., Suarez-Grimalt, R., Vrontou, E., and Lin, A.C. (2020). Localized inhibition in the Drosophila mushroom body. 2020.2003.2026.008300.

Aso, Y., Grubel, K., Busch, S., Friedrich, A.B., Siwanowicz, I., and Tanimoto, H. (2009). The mushroom body of adult Drosophila characterized by GAL4 drivers. J Neurogenet 23, 156-172.

Aso, Y., Hattori, D., Yu, Y., Johnston, R.M., Iyer, N.A., Ngo, T.T., Dionne, H., Abbott, L.F., Axel, R., Tanimoto, H., et al. (2014). The neuronal architecture of the mushroom body provides a logic for associative learning. Elife 3, e04577.

Aso, Y., and Rubin, G.M. (2020). Toward nanoscale localization of memory engrams in Drosophila. J Neurogenet, 1-5.

Athreya, A., Fishkind, D.E., Tang, M., Priebe, C.E., Park, Y., Vogelstein, J.T., Levin, K., Lyzinski, V., and Qin, Y. (2017). Statistical inference on random dot product graphs: a survey. The Journal of Machine Learning Research 18, 8393-8484. 
Babadi, B., and Sompolinsky, H. (2014). Sparseness and expansion in sensory representations. Neuron 83, 1213-1226.

Badel, L., Ohta, K., Tsuchimoto, Y., and Kazama, H. (2016). Decoding of ContextDependent Olfactory Behavior in Drosophila. Neuron 91, 155-167.

Barak, O., Rigotti, M., and Fusi, S. (2013). The Sparseness of Mixed Selectivity Neurons Controls the Generalization-Discrimination Trade-Off. Journal of Neuroscience 33, 38443856.

Bargmann, C.I., and Marder, E. (2013). From the connectome to brain function. Nat Methods 10, 483-490.

Barlow, H.B. (2012). Possible Principles Underlying the Transformations of Sensory Messages. In Sensory Communication (The MIT Press).

Barnstedt, O., Owald, D., Felsenberg, J., Brain, R., Moszynski, J.P., Talbot, C.B., Perrat, P.N., and Waddell, S. (2016). Memory-Relevant Mushroom Body Output Synapses Are Cholinergic. Neuron 89, 1237-1247.

Bates, A.S., Schlegel, P., Roberts, R.J.V., Drummond, N., Tamimi, I.F.M., Turnbull, R., Zhao, X., Marin, E.C., Popovici, P.D., Dhawan, S., et al. (2020). Complete connectomic reconstruction of olfactory projection neurons in the fly brain. 2020.2001.2019.911453.

Bhandawat, V., Olsen, S.R., Gouwens, N.W., Schlief, M.L., and Wilson, R.I. (2007). Sensory processing in the Drosophila antennal lobe increases reliability and separability of ensemble odor representations. Nat Neurosci 10, 1474-1482.

Bopp, R., Macarico da Costa, N., Kampa, B.M., Martin, K.A., and Roth, M.M. (2014). Pyramidal cells make specific connections onto smooth (GABAergic) neurons in mouse visual cortex. PLoS Biol 12, e1001932.

Brown, S.P., and Hestrin, S. (2009). Intracortical circuits of pyramidal neurons reflect their long-range axonal targets. Nature 457, 1133-1136. 
Butcher, N.J., Friedrich, A.B., Lu, Z., Tanimoto, H., and Meinertzhagen, I.A. (2012).

Different classes of input and output neurons reveal new features in microglomeruli of the adult Drosophila mushroom body calyx. J Comp Neurol 520, 2185-2201.

Caron, S.J., Ruta, V., Abbott, L.F., and Axel, R. (2013). Random convergence of olfactory inputs in the Drosophila mushroom body. Nature 497, 113-117.

Cayco-Gajic, N.A., and Silver, R.A. (2019). Re-evaluating Circuit Mechanisms Underlying Pattern Separation. Neuron 101, 584-602.

Chin, S.G., Maguire, S.E., Huoviala, P., Jefferis, G., and Potter, C.J. (2018). Olfactory Neurons and Brain Centers Directing Oviposition Decisions in Drosophila. Cell Rep 24, 1667-1678.

Christiansen, F., Zube, C., Andlauer, T.F., Wichmann, C., Fouquet, W., Owald, D., Mertel, S., Leiss, F., Tavosanis, G., Luna, A.J., et al. (2011). Presynapses in Kenyon cell dendrites in the mushroom body calyx of Drosophila. J Neurosci 31, 9696-9707.

Costa, M., Manton, J.D., Ostrovsky, A.D., Prohaska, S., and Jefferis, G.S.X.E. (2016). NBLAST: Rapid, Sensitive Comparison of Neuronal Structure and Construction of Neuron Family Databases. Neuron 91, 293-311.

Crittenden, J.R., Skoulakis, E.M., Han, K.A., Kalderon, D., and Davis, R.L. (1998). Tripartite mushroom body architecture revealed by antigenic markers. Learn Mem 5, 38-51.

Dana, H., Mohar, B., Sun, Y., Narayan, S., Gordus, A., Hasseman, J.P., Tsegaye, G., Holt, G.T., Hu, A., Walpita, D., et al. (2016). Sensitive red protein calcium indicators for imaging neural activity. Elife 5 .

DasGupta, S., Ferreira, C.H., and Miesenbock, G. (2014). FoxP influences the speed and accuracy of a perceptual decision in Drosophila. Science 344, 901-904.

Dasgupta, S., Stevens, C.F., and Navlakha, S. (2017). A neural algorithm for a fundamental computing problem. Science 358, 793-796. 
Dickinson, M.H., and Muijres, F.T. (2016). The aerodynamics and control of free flight manoeuvres in Drosophila. Philos Trans R Soc Lond B Biol Sci 371.

Dionne, H., Hibbard, K.L., Cavallaro, A., Kao, J.C., and Rubin, G.M. (2018). Genetic Reagents for Making Split-GAL4 Lines in Drosophila. Genetics 209, 31-35.

Dweck, H.K., Ebrahim, S.A., Farhan, A., Hansson, B.S., and Stensmyr, M.C. (2015a). Olfactory proxy detection of dietary antioxidants in Drosophila. Curr Biol 25, 455-466. Dweck, H.K., Ebrahim, S.A., Kromann, S., Bown, D., Hillbur, Y., Sachse, S., Hansson, B.S., and Stensmyr, M.C. (2013). Olfactory preference for egg laying on citrus substrates in Drosophila. Curr Biol 23, 2472-2480.

Dweck, H.K., Ebrahim, S.A., Thoma, M., Mohamed, A.A., Keesey, I.W., Trona, F., LavistaLlanos, S., Svatos, A., Sachse, S., Knaden, M., et al. (2015b). Pheromones mediating copulation and attraction in Drosophila. Proc Natl Acad Sci U S A 112, E2829-2835.

Ebrahim, S.A., Dweck, H.K., Stokl, J., Hofferberth, J.E., Trona, F., Weniger, K., Rybak, J., Seki, Y., Stensmyr, M.C., Sachse, S., et al. (2015). Drosophila Avoids Parasitoids by Sensing Their Semiochemicals via a Dedicated Olfactory Circuit. PLoS Biol 13, e1002318.

Egger, R., Dercksen, V.J., Udvary, D., Hege, H.C., and Oberlaender, M. (2014). Generation of dense statistical connectomes from sparse morphological data. Front Neuroanat 8 , 129.

Eichler, K., Li, F., Litwin-Kumar, A., Park, Y., Andrade, I., Schneider-Mizell, C.M., Saumweber, T., Huser, A., Eschbach, C., Gerber, B., et al. (2017). The complete connectome of a learning and memory centre in an insect brain. Nature 548, 175-182.

Enjin, A., Zaharieva, E.E., Frank, D.D., Mansourian, S., Suh, G.S., Gallio, M., and Stensmyr, M.C. (2016). Humidity Sensing in Drosophila. Curr Biol 26, 1352-1358.

Farris, S.M. (2011). Are mushroom bodies cerebellum-like structures? Arthropod Struct Dev 40, 368-379. 
Felsenberg, J., Jacob, P.F., Walker, T., Barnstedt, O., Edmondson-Stait, A.J., Pleijzier, M.W., Otto, N., Schlegel, P., Sharifi, N., Perisse, E., et al. (2018). Integration of Parallel Opposing Memories Underlies Memory Extinction. Cell 175, 709-722 e715.

Frank, D.D., Jouandet, G.C., Kearney, P.J., Macpherson, L.J., and Gallio, M. (2015).

Temperature representation in the Drosophila brain. Nature 519, 358-361.

Groschner, L.N., and Miesenbock, G. (2019). Mechanisms of Sensory Discrimination: Insights from Drosophila Olfaction. Annu Rev Biophys 48, 209-229.

Grosjean, Y., Rytz, R., Farine, J.P., Abuin, L., Cortot, J., Jefferis, G.S., and Benton, R. (2011). An olfactory receptor for food-derived odours promotes male courtship in Drosophila. Nature 478, 236-240.

Gruntman, E., and Turner, G.C. (2013). Integration of the olfactory code across dendritic claws of single mushroom body neurons. Nat Neurosci 16, 1821-1829.

Guven-Ozkan, T., and Davis, R.L. (2014). Functional neuroanatomy of Drosophila olfactory memory formation. Learn Mem 21, 519-526.

Hallem, E.A., and Carlson, J.R. (2006). Coding of odors by a receptor repertoire. Cell 125, 143-160.

Hamada, F.N., Rosenzweig, M., Kang, K., Pulver, S.R., Ghezzi, A., Jegla, T.J., and Garrity, P.A. (2008). An internal thermal sensor controlling temperature preference in Drosophila. Nature 454, 217-220.

Hansel, D., and van Vreeswijk, C. (2012). The mechanism of orientation selectivity in primary visual cortex without a functional map. J Neurosci 32, 4049-4064.

Heisenberg, M. (2003). Mushroom body memoir: from maps to models. Nat Rev Neurosci 4, 266-275.

Hige, T., Aso, Y., Rubin, G.M., and Turner, G.C. (2015). Plasticity-driven individualization of olfactory coding in mushroom body output neurons. Nature 526, 258-262. 
Hussain, A., Zhang, M., Ucpunar, H.K., Svensson, T., Quillery, E., Gompel, N., Ignell, R., and Grunwald Kadow, I.C. (2016). Ionotropic Chemosensory Receptors Mediate the Taste and Smell of Polyamines. PLoS Biol 14, e1002454.

Inada, K., Tsuchimoto, Y., and Kazama, H. (2017). Origins of Cell-Type-Specific Olfactory Processing in the Drosophila Mushroom Body Circuit. Neuron 95, 357-367 e354.

Ito, K., Awano, W., Suzuki, K., Hiromi, Y., and Yamamoto, D. (1997). The Drosophila mushroom body is a quadruple structure of clonal units each of which contains a virtually identical set of neurones and glial cells. Development 124, 761-771. Jefferis, G.S., Potter, C.J., Chan, A.M., Marin, E.C., Rohlfing, T., Maurer, C.R., Jr., and Luo, L. (2007). Comprehensive maps of Drosophila higher olfactory centers: spatially segregated fruit and pheromone representation. Cell 128, 1187-1203.

Jonas, E., and Kording, K. (2015). Automatic discovery of cell types and microcircuitry from neural connectomics. Elife 4, e04250.

Kasthuri, N., Hayworth, K.J., Berger, D.R., Schalek, R.L., Conchello, J.A., Knowles-Barley, S., Lee, D., Vazquez-Reina, A., Kaynig, V., Jones, T.R., et al. (2015). Saturated Reconstruction of a Volume of Neocortex. Cell 162, 648-661.

Klapoetke, N.C., Murata, Y., Kim, S.S., Pulver, S.R., Birdsey-Benson, A., Cho, Y.K., Morimoto, T.K., Chuong, A.S., Carpenter, E.J., Tian, Z., et al. (2014). Independent optical excitation of distinct neural populations. Nat Methods 11, 338-346.

Knaden, M., Strutz, A., Ahsan, J., Sachse, S., and Hansson, B.S. (2012). Spatial representation of odorant valence in an insect brain. Cell Rep 1, 392-399.

Kornfeld, J., and Denk, W. (2018). Progress and remaining challenges in high-throughput volume electron microscopy. Curr Opin Neurobiol 50, 261-267.

Koulakov, A.A., Kolterman, B.E., Enikolopov, A.G., and Rinberg, D. (2011). In search of the structure of human olfactory space. Front Syst Neurosci 5, 65. 
Kremer, M.C., Christiansen, F., Leiss, F., Paehler, M., Knapek, S., Andlauer, T.F., Forstner, F., Kloppenburg, P., Sigrist, S.J., and Tavosanis, G. (2010). Structural long-term changes at mushroom body input synapses. Curr Biol 20, 1938-1944.

Kurtovic, A., Widmer, A., and Dickson, B.J. (2007). A single class of olfactory neurons mediates behavioural responses to a Drosophila sex pheromone. Nature 446, 542-546. Laissue, P.P., and Vosshall, L.B. (2008). The olfactory sensory map in Drosophila. Adv Exp Med Biol 628, 102-114.

Laughlin, S. (1981). A simple coding procedure enhances a neuron's information capacity. Z Naturforsch C Biosci 36, 910-912.

Lebreton, S., Borrero-Echeverry, F., Gonzalez, F., Solum, M., Wallin, E.A., Hedenstrom, E., Hansson, B.S., Gustavsson, A.L., Bengtsson, M., Birgersson, G., et al. (2017). A Drosophila female pheromone elicits species-specific long-range attraction via an olfactory channel with dual specificity for sex and food. BMC Biol 15, 88.

Lee, T., Lee, A., and Luo, L. (1999). Development of the Drosophila mushroom bodies: sequential generation of three distinct types of neurons from a neuroblast. Development 126, 4065-4076.

Lee, W.C., Bonin, V., Reed, M., Graham, B.J., Hood, G., Glattfelder, K., and Reid, R.C. (2016). Anatomy and function of an excitatory network in the visual cortex. Nature 532, 370-374.

Leiss, F., Groh, C., Butcher, N.J., Meinertzhagen, I.A., and Tavosanis, G. (2009). Synaptic organization in the adult Drosophila mushroom body calyx. J Comp Neurol 517, 808-824. Leitch, B., and Laurent, G. (1996). GABAergic synapses in the antennal lobe and mushroom body of the locust olfactory system. J Comp Neurol 372, 487-514. 
Li, P.H., Lindsey, L.F., Januszewski, M., Zheng, Z., Bates, A.S., Taisz, I., Tyka, M., Nichols, M., Li, F., Perlman, E., et al. (2019). Automated Reconstruction of a Serial-Section EM Drosophila Brain with Flood-Filling Networks and Local Realignment. 605634. Lichtman, J.W., and Sanes, J.R. (2008). Ome sweet ome: what can the genome tell us about the connectome? Curr Opin Neurobiol 18, 346-353.

Lin, A.C., Bygrave, A.M., de Calignon, A., Lee, T., and Miesenbock, G. (2014). Sparse, decorrelated odor coding in the mushroom body enhances learned odor discrimination. Nat Neurosci 17, 559-568.

Lin, H.H., Lai, J.S., Chin, A.L., Chen, Y.C., and Chiang, A.S. (2007). A map of olfactory representation in the Drosophila mushroom body. Cell 128, 1205-1217.

Litwin-Kumar, A., Harris, K.D., Axel, R., Sompolinsky, H., and Abbott, L.F. (2017). Optimal Degrees of Synaptic Connectivity. Neuron 93, 1153-1164 e1157.

Litwin-Kumar, A., and Turaga, S.C. (2019). Constraining computational models using electron microscopy wiring diagrams. Curr Opin Neurobiol 58, 94-100.

Liu, Q., Yang, X., Tian, J., Gao, Z., Wang, M., Li, Y., and Guo, A. (2016). Gap junction networks in mushroom bodies participate in visual learning and memory in Drosophila. Elife 5.

Liu, W.W., Mazor, O., and Wilson, R.I. (2015). Thermosensory processing in the Drosophila brain. Nature 519, 353-357.

Liu, X., and Davis, R.L. (2009). The GABAergic anterior paired lateral neuron suppresses and is suppressed by olfactory learning. Nat Neurosci 12, 53-59.

MacQueen, J. (1967). Some methods for classification and analysis of multivariate observations. Paper presented at: Proceedings of the fifth Berkeley symposium on mathematical statistics and probability (Oakland, CA, USA). 
Mansourian, S., and Stensmyr, M.C. (2015). The chemical ecology of the fly. Curr Opin Neurobiol 34, 95-102.

Marr, D. (1969). A theory of cerebellar cortex. J Physiol 202, 437-470.

Meinertzhagen, I.A. (2010). The organisation of invertebrate brains: cells, synapses and circuits. 91, 64-71.

Meinertzhagen, I.A. (2018). Of what use is connectomics? A personal perspective on the Drosophila connectome. J Exp Biol 221.

Min, S., Ai, M., Shin, S.A., and Suh, G.S. (2013). Dedicated olfactory neurons mediating attraction behavior to ammonia and amines in Drosophila. Proc Natl Acad Sci U S A 110, E1321-1329.

Mishchenko, Y., Hu, T., Spacek, J., Mendenhall, J., Harris, K.M., and Chklovskii, D.B. (2010). Ultrastructural Analysis of Hippocampal Neuropil from the Connectomics Perspective. Neuron 67, 1009-1020.

Murtagh, F., and Legendre, P. (2014). Ward's Hierarchical Agglomerative Clustering Method: Which Algorithms Implement Ward's Criterion? Journal of Classification 31, 274-295.

Murthy, M., Fiete, I., and Laurent, G. (2008). Testing odor response stereotypy in the Drosophila mushroom body. Neuron 59, 1009-1023.

Nern, A., Pfeiffer, B.D., and Rubin, G.M. (2015). Optimized tools for multicolor stochastic labeling reveal diverse stereotyped cell arrangements in the fly visual system. Proc Natl Acad Sci U S A 112, E2967-2976.

Newman, M. (2018). 7. Measures and metrics. In Networks (Oxford University Press).

Ofstad, T.A., Zuker, C.S., and Reiser, M.B. (2011). Visual place learning in Drosophila melanogaster. Nature 474, 204-207. 
Olsen, S.R., and Wilson, R.I. (2008). Lateral presynaptic inhibition mediates gain control in an olfactory circuit. Nature $452,956-960$.

Owald, D., and Waddell, S. (2015). Olfactory learning skews mushroom body output pathways to steer behavioral choice in Drosophila. Curr Opin Neurobiol 35, 178-184. Pehlevan, C., Genkin, A., and Chklovskii, D.B. (2017). A clustering neural network model of insect olfaction. Paper presented at: Signals, Systems, and Computers, 2017 51st Asilomar Conference on (IEEE).

Perisse, E., Burke, C., Huetteroth, W., and Waddell, S. (2013). Shocking revelations and saccharin sweetness in the study of Drosophila olfactory memory. Curr Biol 23, R752763.

Perisse, E., Owald, D., Barnstedt, O., Talbot, C.B., Huetteroth, W., and Waddell, S. (2016). Aversive Learning and Appetitive Motivation Toggle Feed-Forward Inhibition in the Drosophila Mushroom Body. Neuron 90, 1086-1099.

Rigotti, M., Barak, O., Warden, M.R., Wang, X.J., Daw, N.D., Miller, E.K., and Fusi, S. (2013). The importance of mixed selectivity in complex cognitive tasks. Nature 497, 585590.

Ronderos, D.S., Lin, C.C., Potter, C.J., and Smith, D.P. (2014). Farnesol-detecting olfactory neurons in Drosophila. J Neurosci 34, 3959-3968.

Root, C.M., Semmelhack, J.L., Wong, A.M., Flores, J., and Wang, J.W. (2007).

Propagation of olfactory information in Drosophila. Proc Natl Acad Sci U S A 104, 1182611831.

Scheffer, L.K., Xu, C.S., Januszewski, M., Lu, Z., Takemura, S.-y., Hayworth, K.J., Huang, G.B., Shinomiya, K., Maitin-Shepard, J., Berg, S., et al. (2020). A Connectome and Analysis of the Adult <em>Drosophila</em> Central Brain. 2020.2004.2007.030213. 
Schlief, M.L., and Wilson, R.I. (2007). Olfactory processing and behavior downstream from highly selective receptor neurons. Nat Neurosci 10, 623-630.

Schneider-Mizell, C.M., Gerhard, S., Longair, M., Kazimiers, T., Li, F., Zwart, M.F., Champion, A., Midgley, F.M., Fetter, R.D., Saalfeld, S., et al. (2016). Quantitative neuroanatomy for connectomics in Drosophila. Elife 5.

Schubert, M., Hansson, B.S., and Sachse, S. (2014). The banana code-natural blend processing in the olfactory circuitry of Drosophila melanogaster. Front Physiol 5, 59. Schürmann, F.-W. (1974). Bemerkungen zur funktion der corpora pedunculata im gehirn der insekten aus morphologischer sicht. Experimental Brain Research 19, 406-432.

Seki, Y., Dweck, H.K.M., Rybak, J., Wicher, D., Sachse, S., and Hansson, B.S. (2017). Olfactory coding from the periphery to higher brain centers in the Drosophila brain. BMC Biol 15, 56.

Semmelhack, J.L., and Wang, J.W. (2009). Select Drosophila glomeruli mediate innate olfactory attraction and aversion. Nature 459, 218-223.

Shomrat, T., Turchetti-Maia, A.L., Stern-Mentch, N., Basil, J.A., and Hochner, B. (2015). The vertical lobe of cephalopods: an attractive brain structure for understanding the evolution of advanced learning and memory systems. J Comp Physiol A Neuroethol Sens Neural Behav Physiol 201, 947-956.

Silbering, A.F., Rytz, R., Grosjean, Y., Abuin, L., Ramdya, P., Jefferis, G.S., and Benton, R. (2011). Complementary function and integrated wiring of the evolutionarily distinct Drosophila olfactory subsystems. J Neurosci 31, 13357-13375.

Sporns, O., and Betzel, R.F. (2016). Modular Brain Networks. Annu Rev Psychol 67, 613640. 
Stensmyr, M.C., Dweck, H.K., Farhan, A., Ibba, I., Strutz, A., Mukunda, L., Linz, J., Grabe, V., Steck, K., Lavista-Llanos, S., et al. (2012). A conserved dedicated olfactory circuit for detecting harmful microbes in Drosophila. Cell 151, 1345-1357.

Stevens, C.F. (2015). What the fly's nose tells the fly's brain. Proc Natl Acad Sci U S A $112,9460-9465$.

Stocker, R.F., Lienhard, M.C., Borst, A., and Fischbach, K.F. (1990). Neuronal architecture of the antennal lobe in Drosophila melanogaster. Cell Tissue Res 262, 9-34.

Sugie, A., Marchetti, G., and Tavosanis, G. (2018). Structural aspects of plasticity in the nervous system of Drosophila. Neural Dev 13, 14.

Suh, G.S., Wong, A.M., Hergarden, A.C., Wang, J.W., Simon, A.F., Benzer, S., Axel, R., and Anderson, D.J. (2004). A single population of olfactory sensory neurons mediates an innate avoidance behaviour in Drosophila. Nature 431, 854-859.

Takemura, S.Y., Aso, Y., Hige, T., Wong, A., Lu, Z., Xu, C.S., Rivlin, P.K., Hess, H., Zhao, T., Parag, T., et al. (2017). A connectome of a learning and memory center in the adult Drosophila brain. Elife 6 .

Tanaka, N.K., Awasaki, T., Shimada, T., and Ito, K. (2004). Integration of chemosensory pathways in the Drosophila second-order olfactory centers. Curr Biol 14, 449-457.

Tanaka, N.K., Tanimoto, H., and Ito, K. (2008). Neuronal assemblies of the Drosophila mushroom body. J Comp Neurol 508, 711-755.

Technau, G., and Heisenberg, M. (1982). Neural reorganization during metamorphosis of the corpora pedunculata in Drosophila melanogaster. Nature 295, 405-407.

Tsakiris, A., Koutinas, A.A., Psarianos, C., Kourkoutas, Y., and Bekatorou, A. (2010). A new process for wine production by penetration of yeast in uncrushed frozen grapes. Appl Biochem Biotechnol 162, 1109-1121. 
Turner, G.C., Bazhenov, M., and Laurent, G. (2008). Olfactory representations by Drosophila mushroom body neurons. J Neurophysiol 99, 734-746. van der Goes van Naters, W., and Carlson, J.R. (2007). Receptors and neurons for fly odors in Drosophila. Curr Biol 17, 606-612.

Venken, K.J., Simpson, J.H., and Bellen, H.J. (2011). Genetic manipulation of genes and cells in the nervous system of the fruit fly. Neuron 72, 202-230.

Wong, A.M., Wang, J.W., and Axel, R. (2002). Spatial representation of the glomerular map in the Drosophila protocerebrum. Cell 109, 229-241.

Yagi, R., Mabuchi, Y., Mizunami, M., and Tanaka, N.K. (2016). Convergence of multimodal sensory pathways to the mushroom body calyx in Drosophila melanogaster. Sci Rep 6, 29481.

Yasuyama, K., Meinertzhagen, I.A., and Schurmann, F.W. (2002). Synaptic organization of the mushroom body calyx in Drosophila melanogaster. J Comp Neurol 445, 211-226. Zhang, X., Coates, K., Dacks, A., Gunay, C., Lauritzen, J.S., Li, F., Calle-Schuler, S.A., Bock, D., and Gaudry, Q. (2019). Local synaptic inputs support opposing, network-specific odor representations in a widely projecting modulatory neuron. Elife 8.

Zheng, Z., Lauritzen, J.S., Perlman, E., Robinson, C.G., Nichols, M., Milkie, D., Torrens, O., Price, J., Fisher, C.B., Sharifi, N., et al. (2018). A Complete Electron Microscopy Volume of the Brain of Adult Drosophila melanogaster. Cell 174, 730-743 e722. 
Figure 1. Structured olfactory input to the mushroom body.

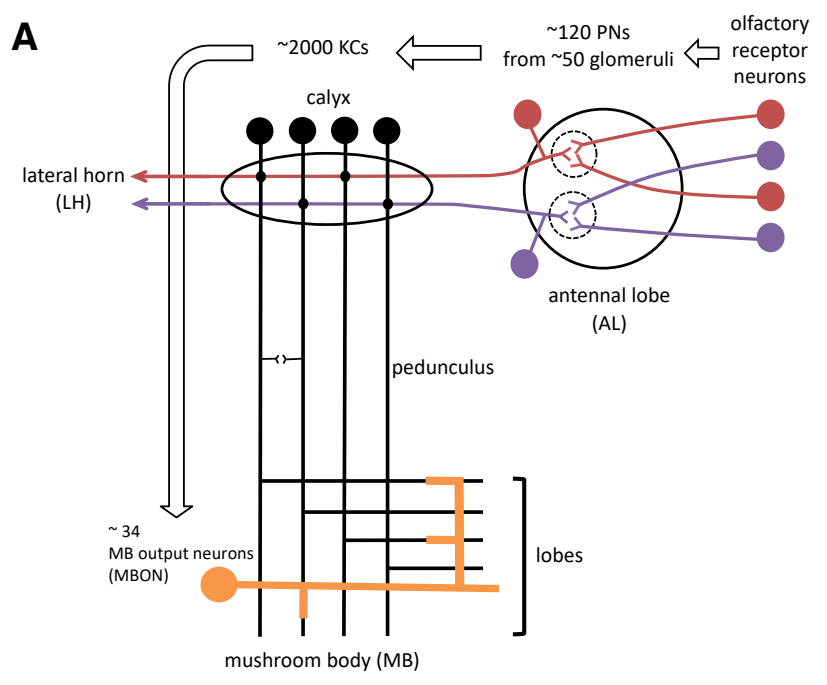

C
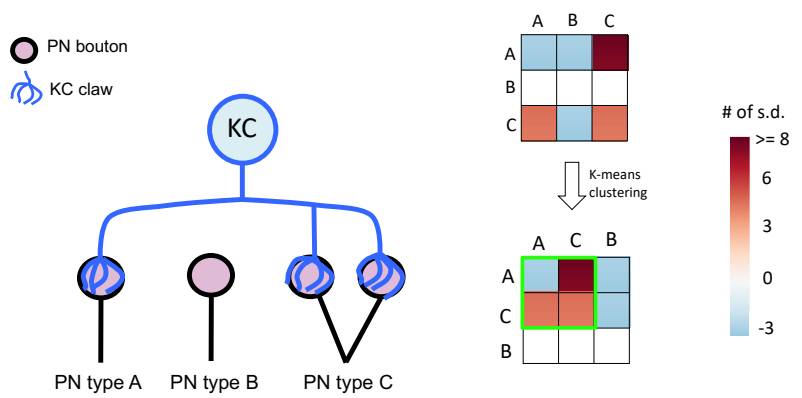

z-score $=\frac{\text { observed }- \text { mean (null model) }}{\text { s.d. (null model) }}$

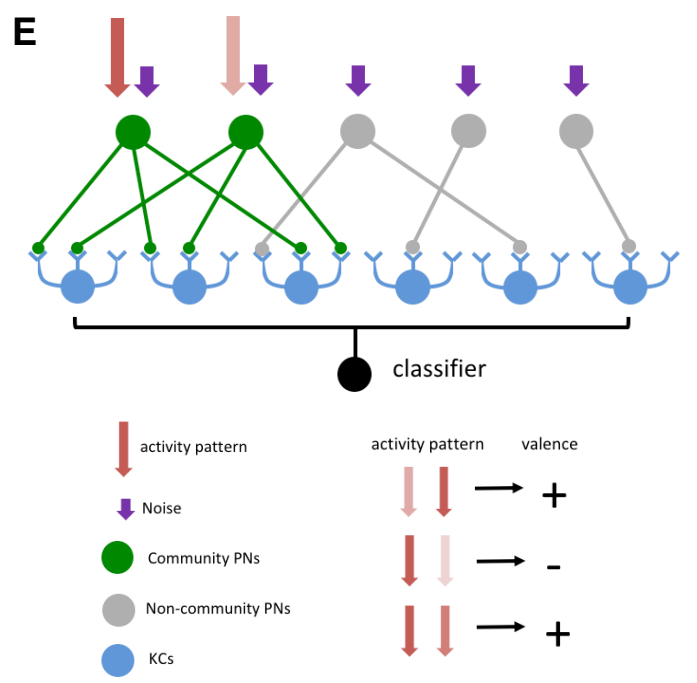

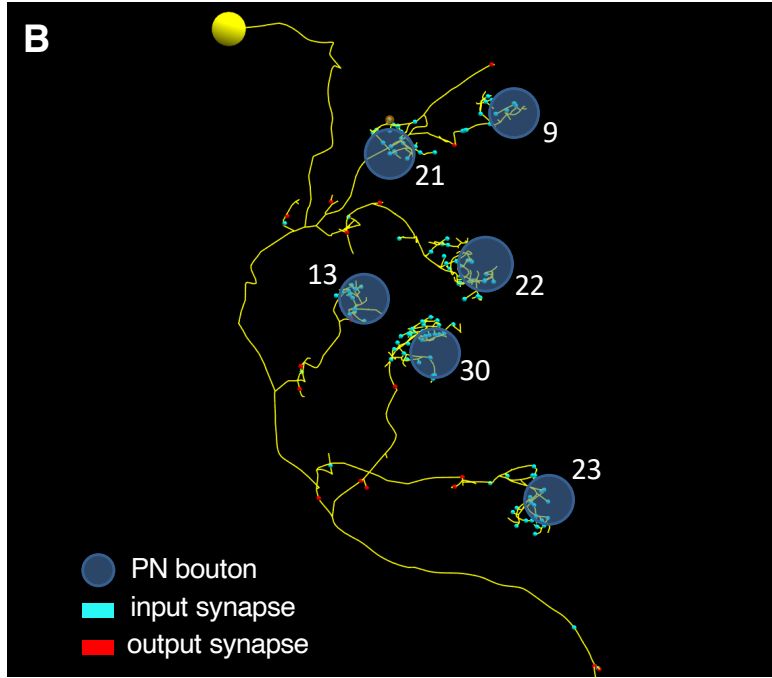

D observed vs. random bouton model

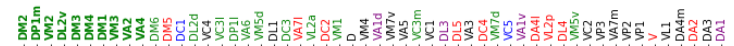

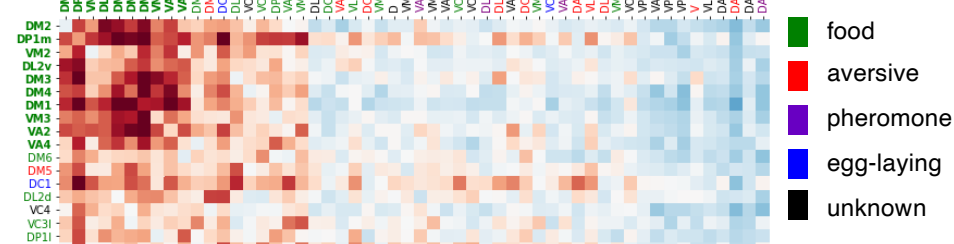

$\mathbf{F}$

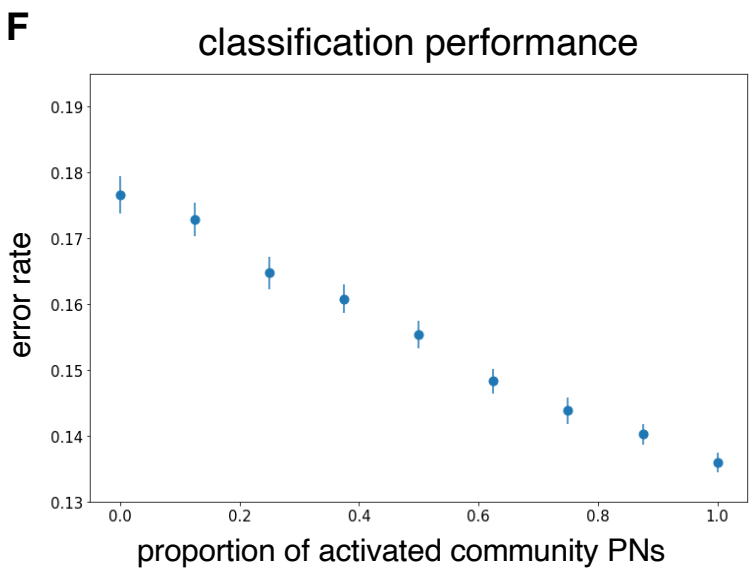


Figure 2. Biased sampling of community PNs by KCs.

A random bouton model vs. postsynaptic claw counts

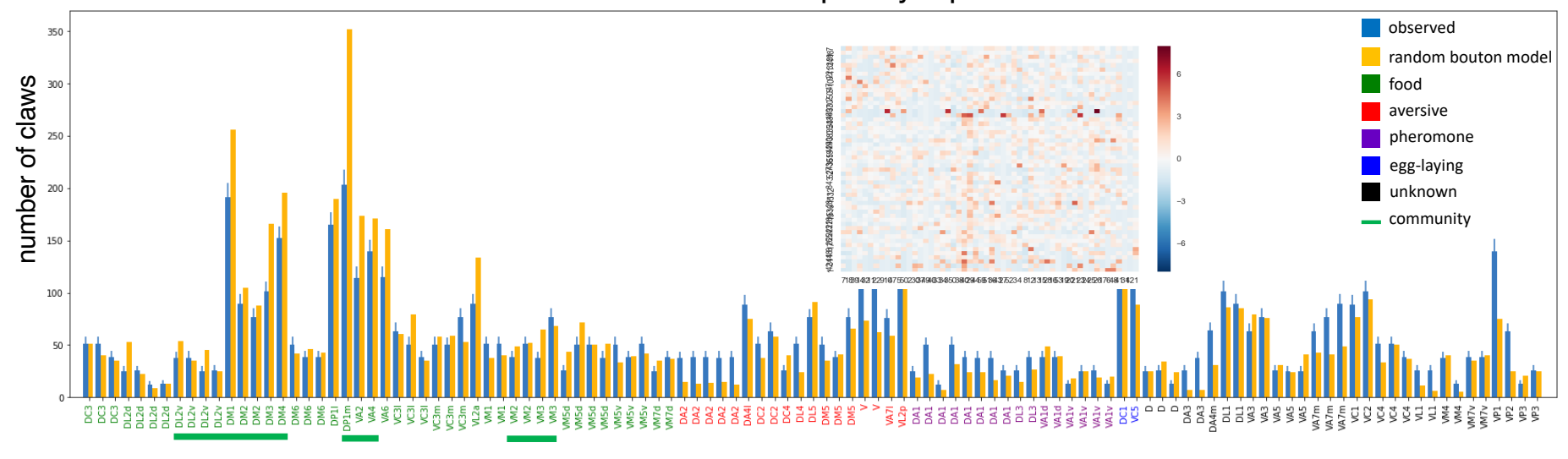

B

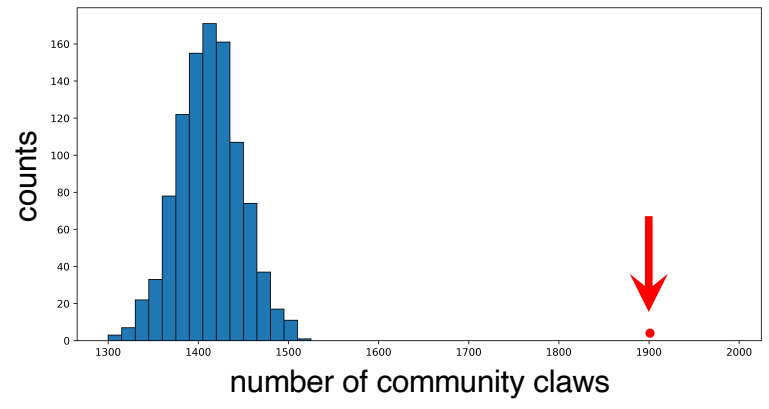

C

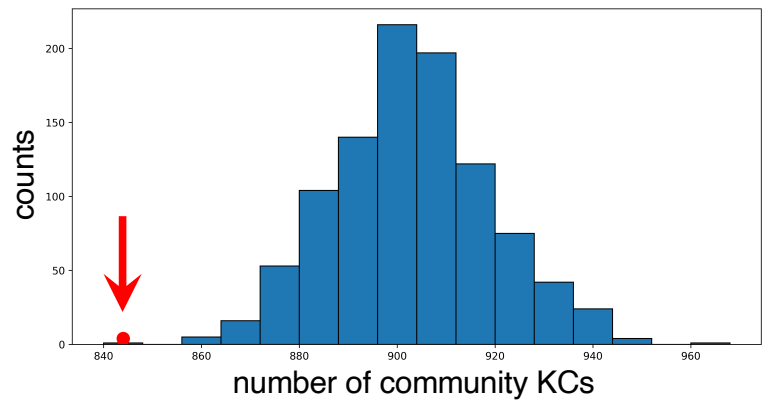

D observed vs. random claw model

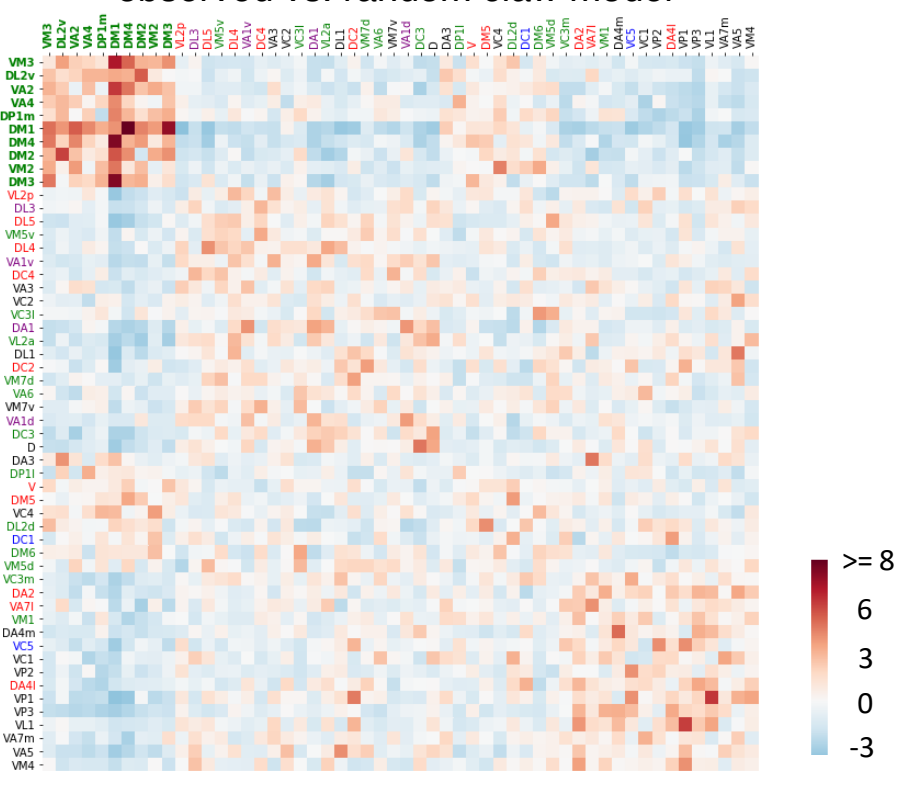


Figure 3. Over-convergence of community PNs to KCs.

A

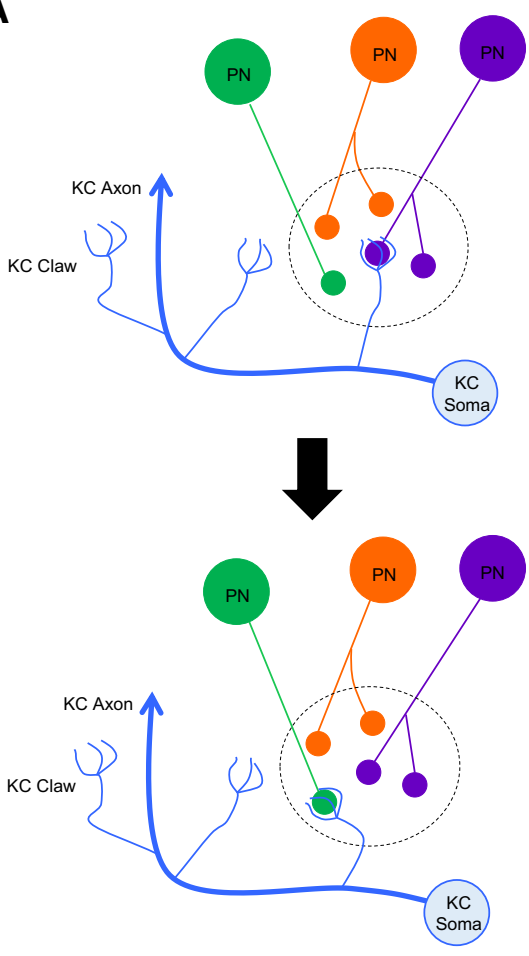

B

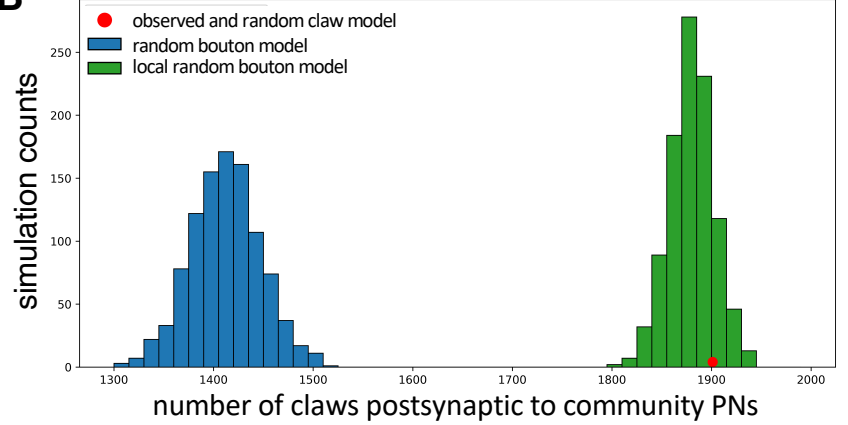

C

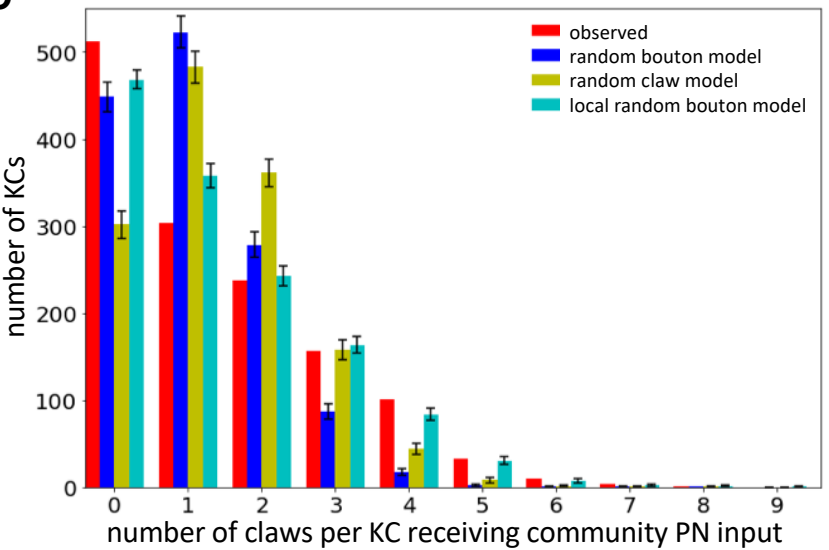

D

local random bouton vs. random bouton model

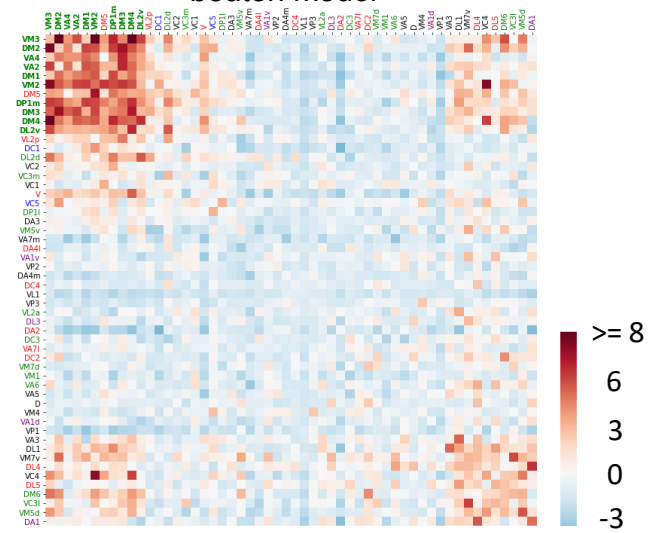

E

observed vs. local random bouton model

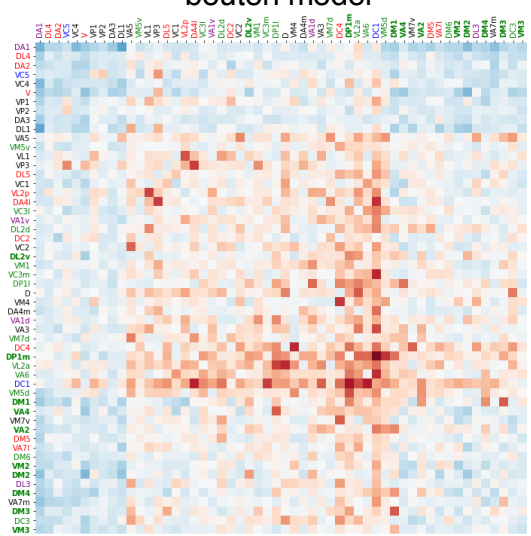

$\mathbf{F}$

observed vs. local random bouton model (Fig1D order)

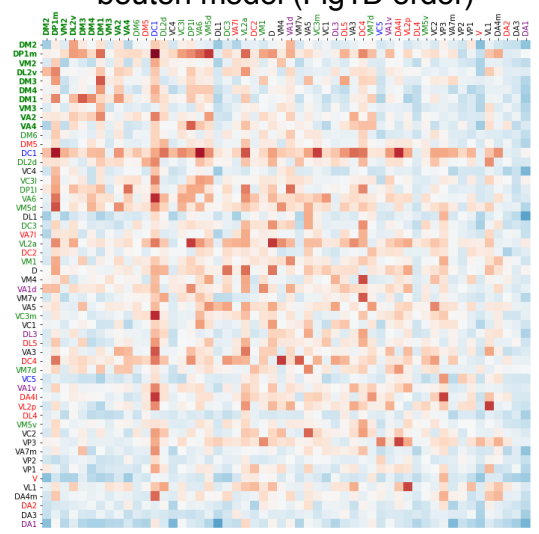


Figure 4. Arbor overlap between subsets of community PNs and KCs.
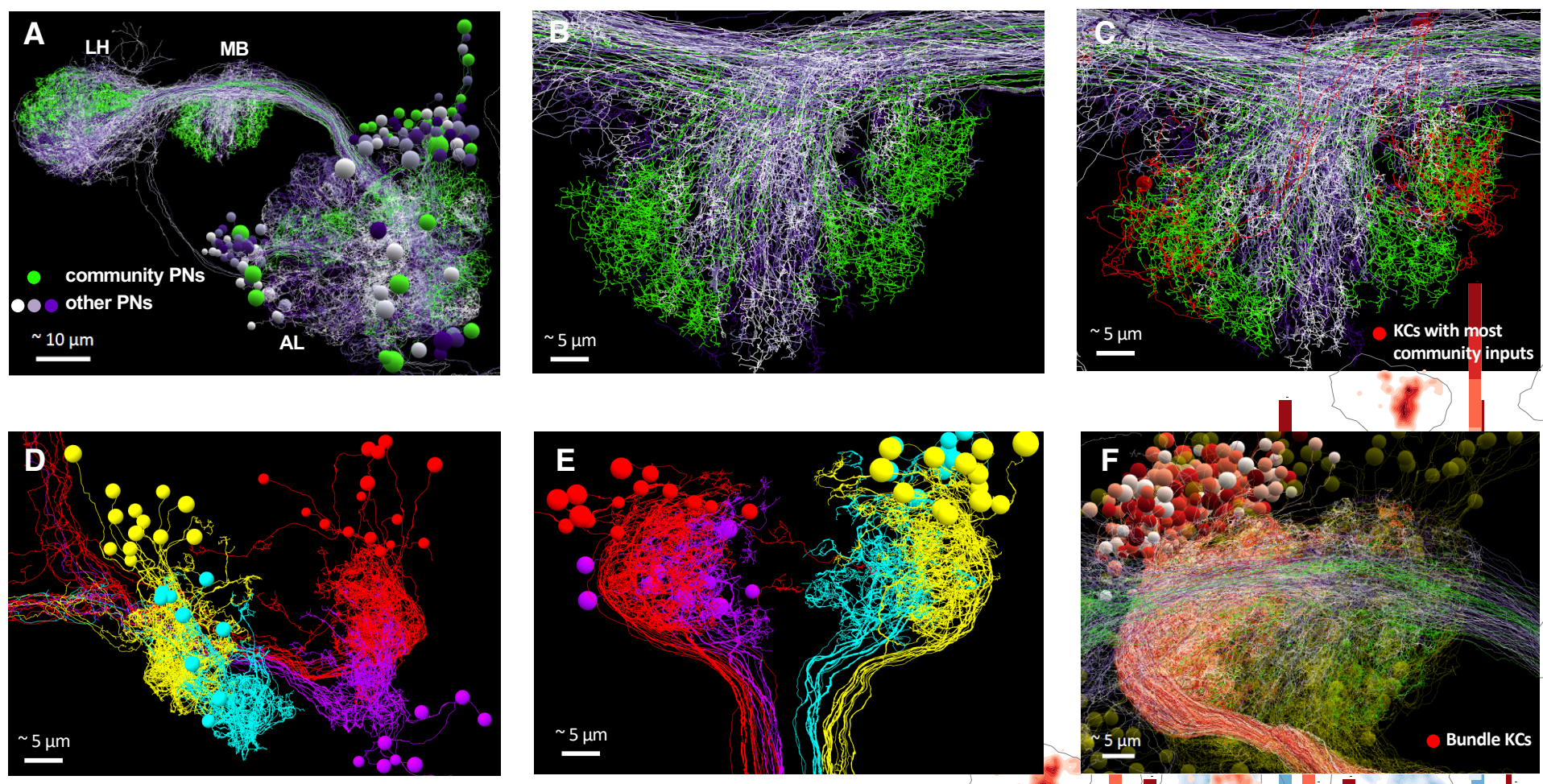

G

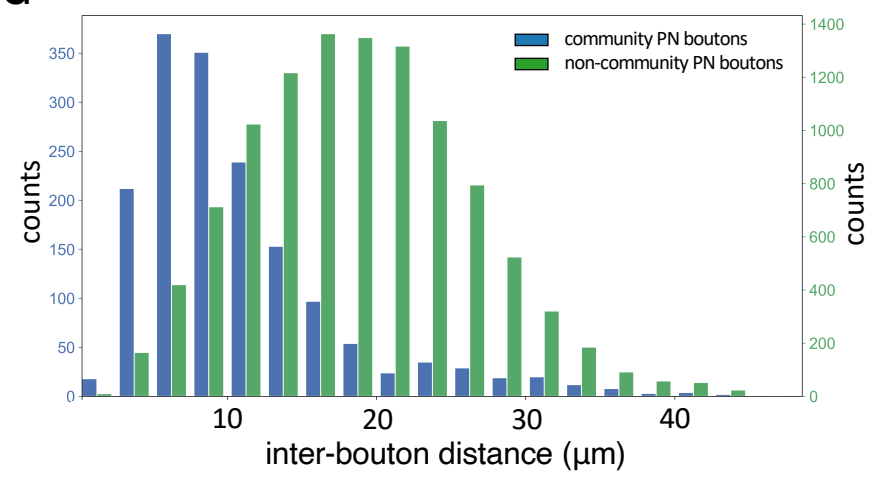

H

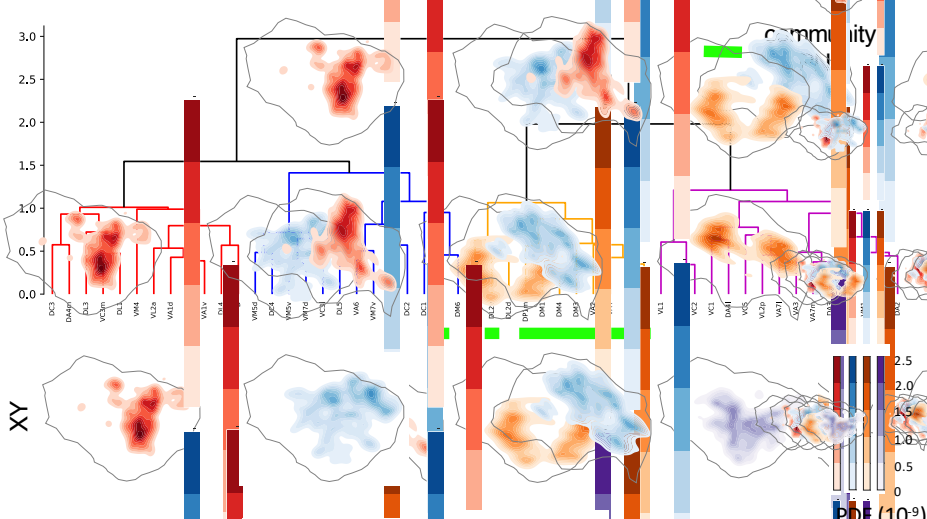

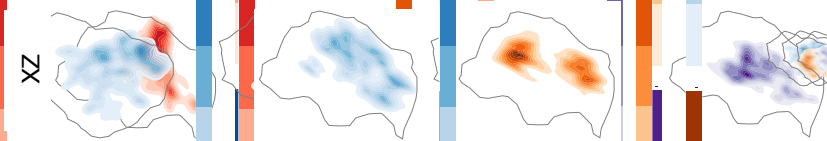


Supplemental Figure 1.

A

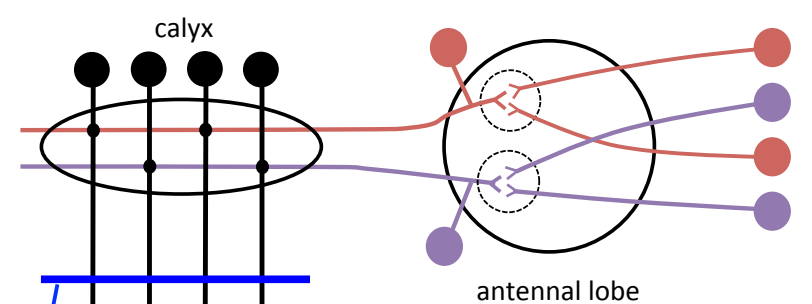

(AL)
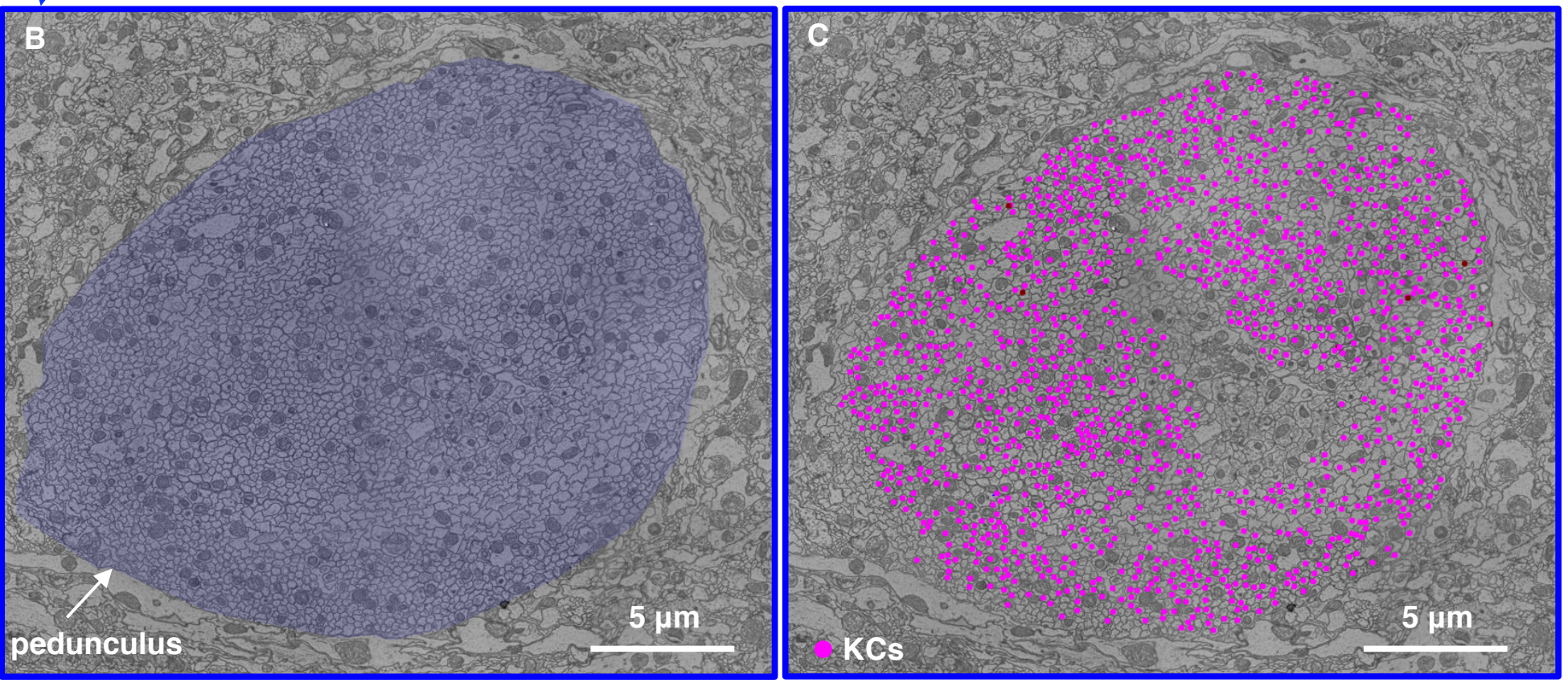


\section{Supplemental Figure 2.}

A

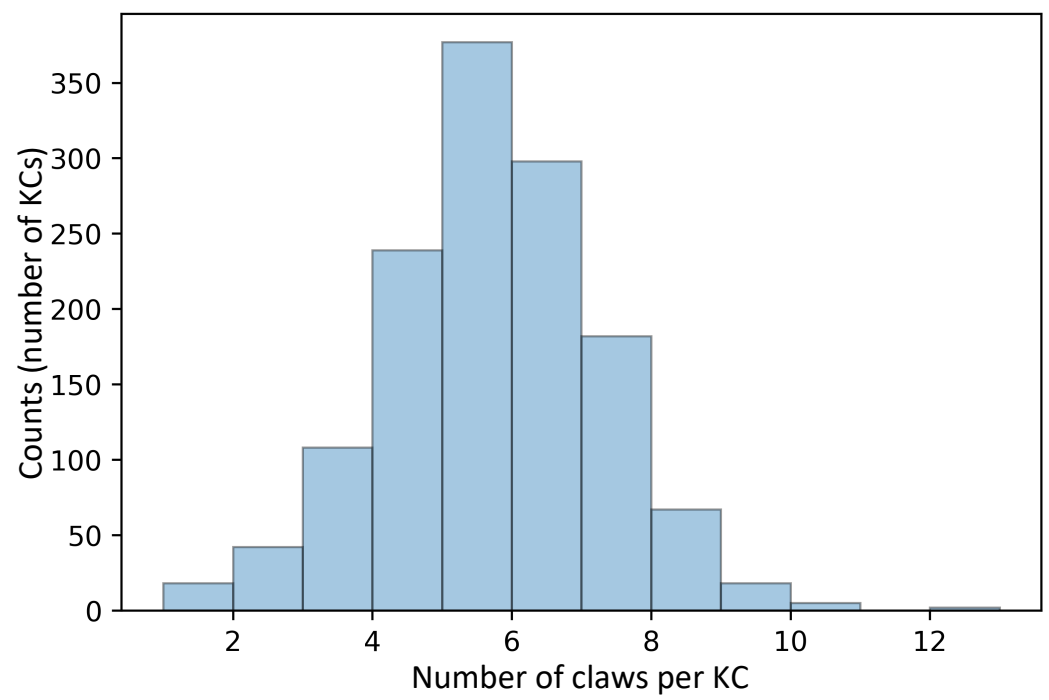

B

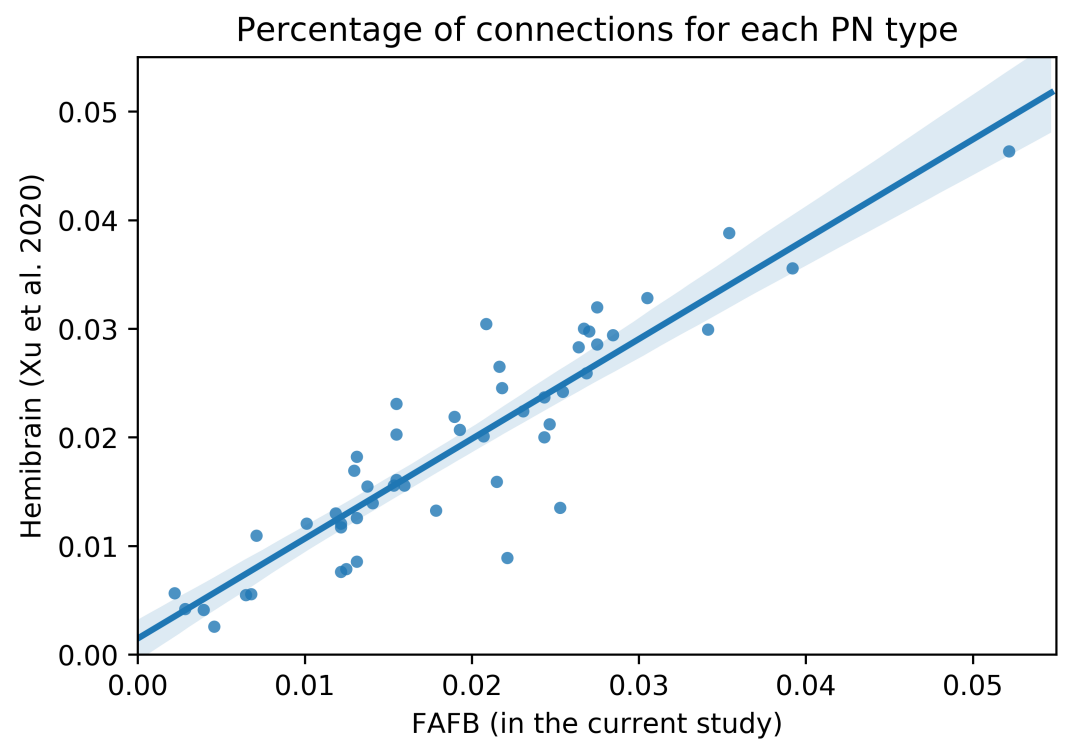

C

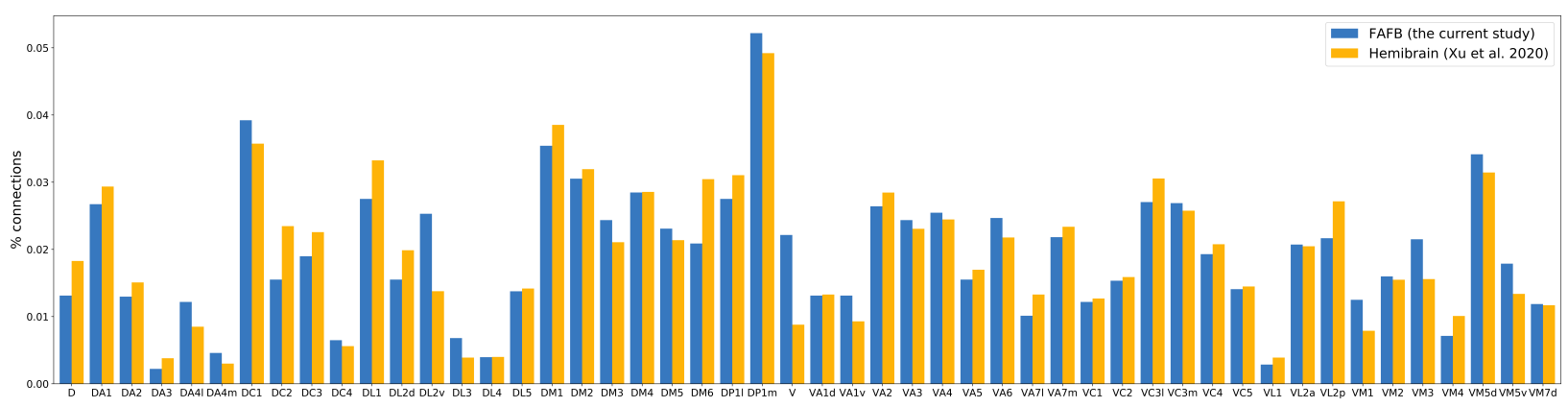

PN type 


\section{Supplemental Figure 3.}

A

A

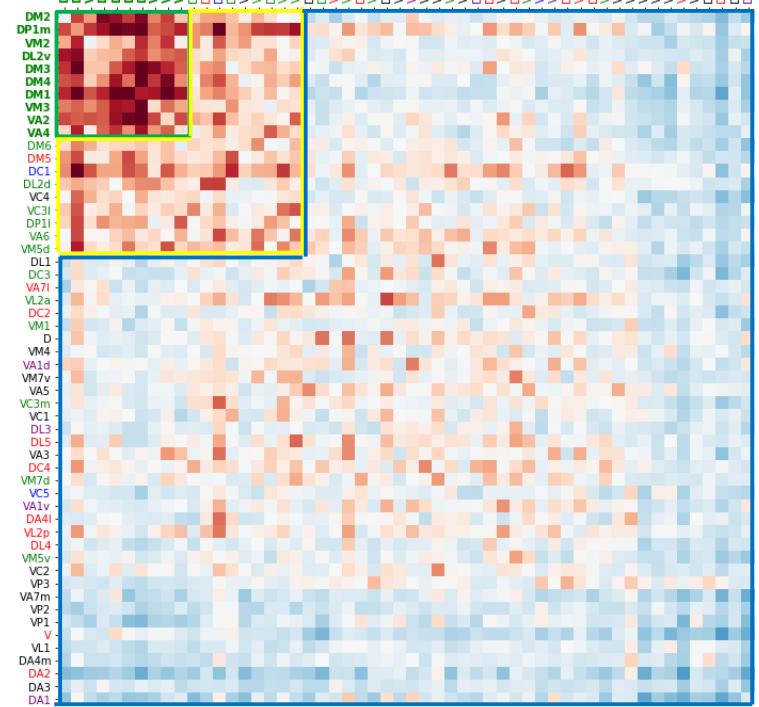

C

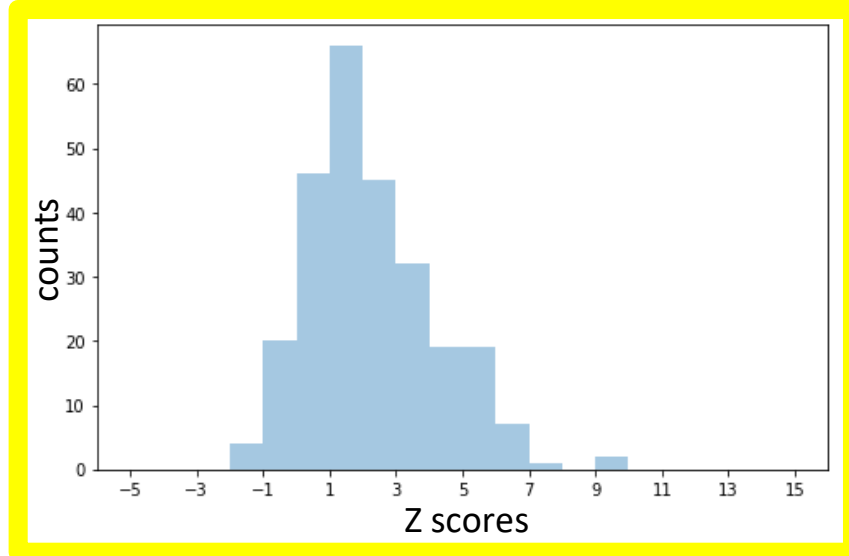

B

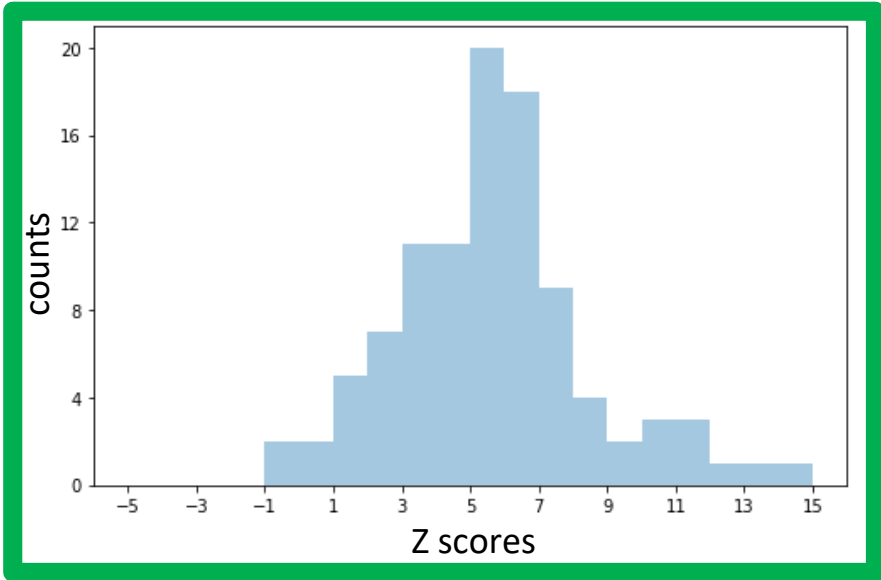

D

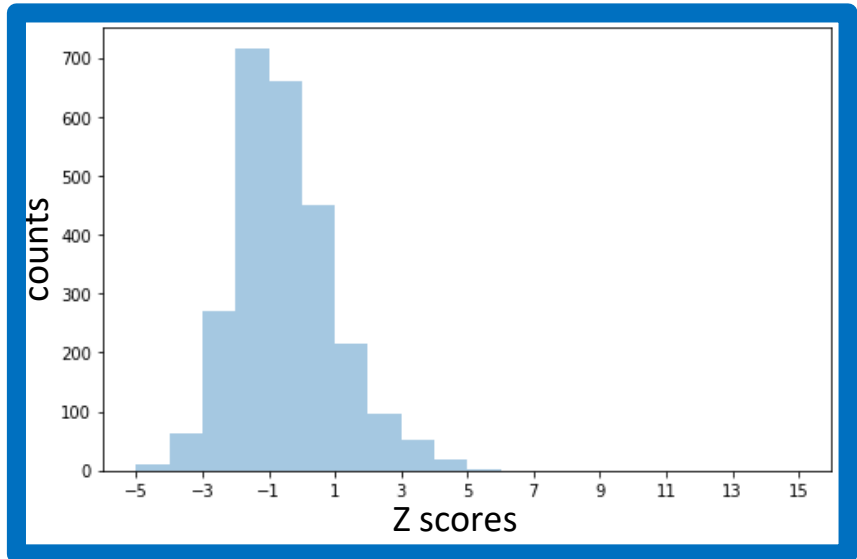

E

randomized and reclustered

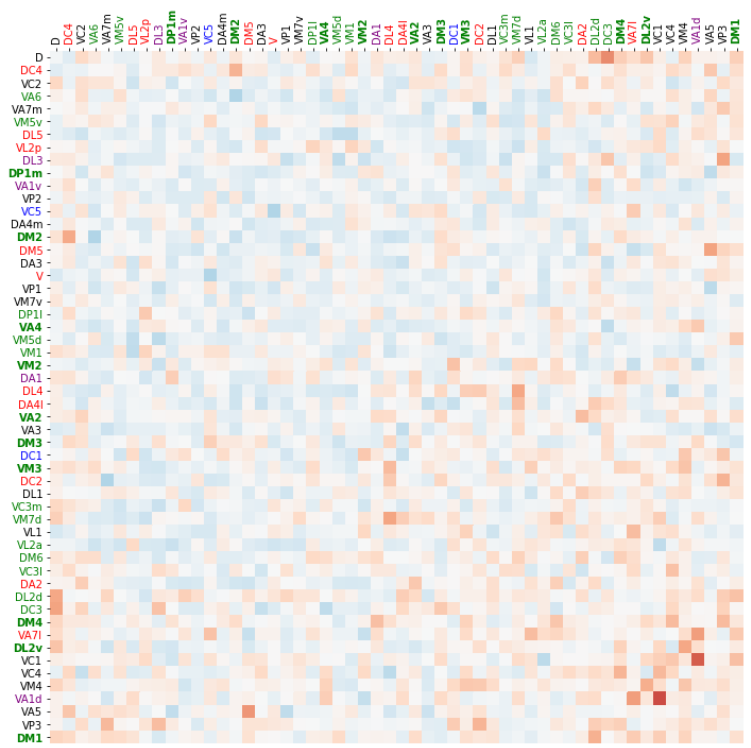


Supplemental Figure 4

Fig 1D clustering order

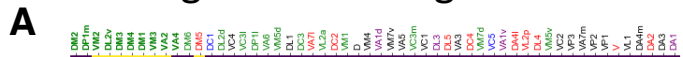

$P<0.05$
$P>=0.05$

Bouton-claw connection counts

Synapse counts

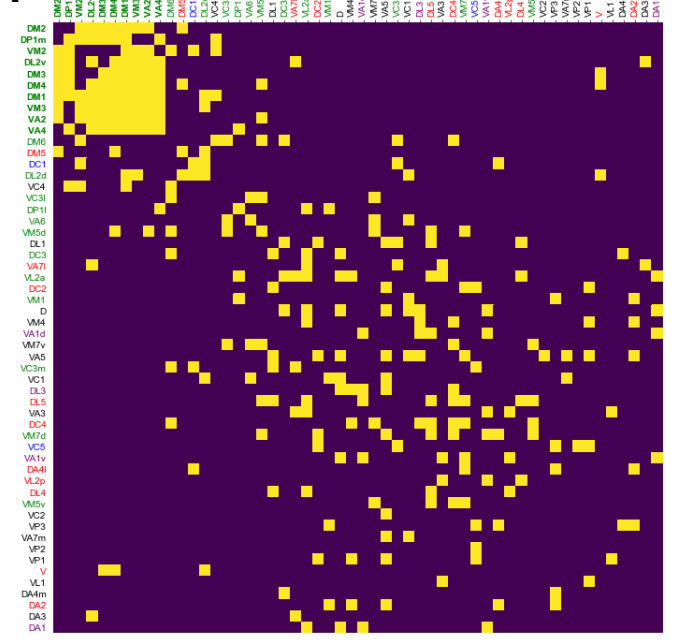

C

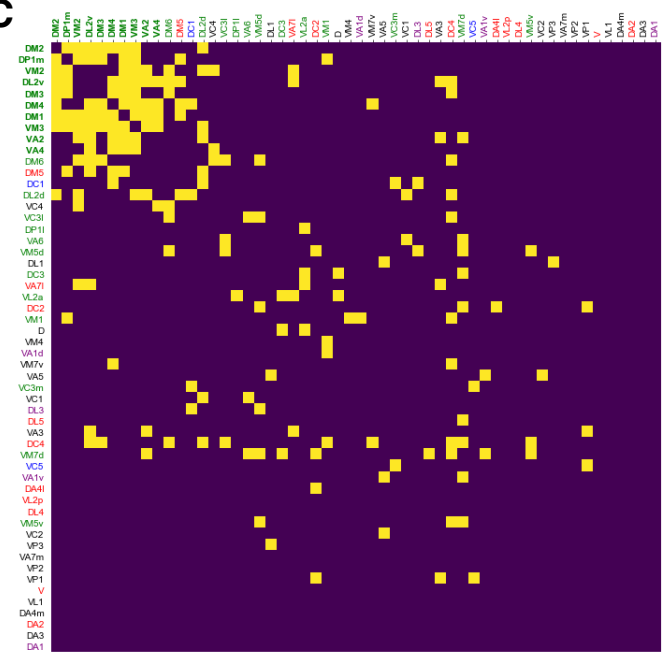

E

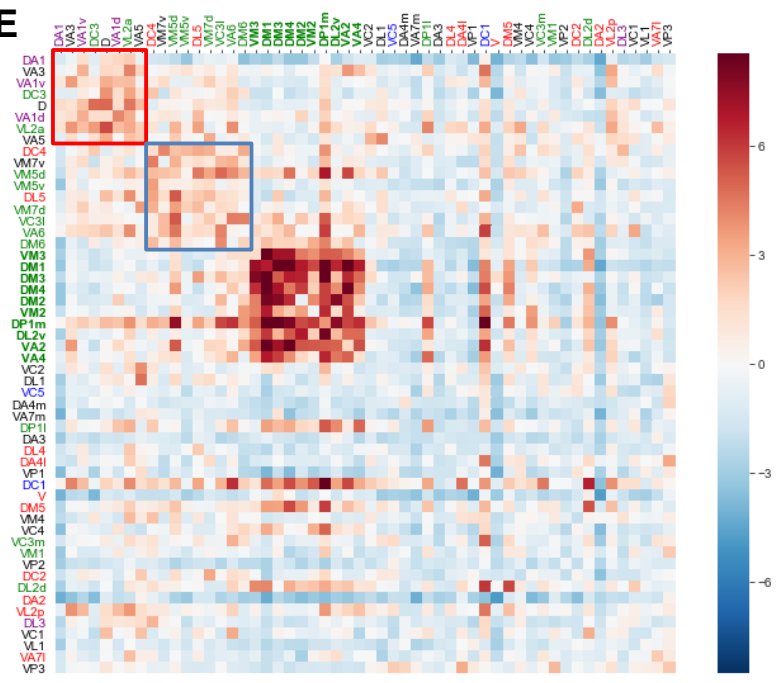

Re-cluster

B

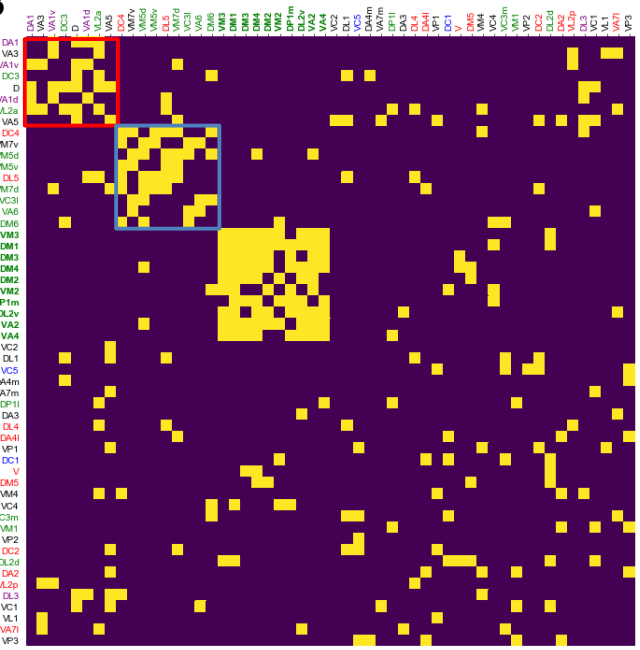

D
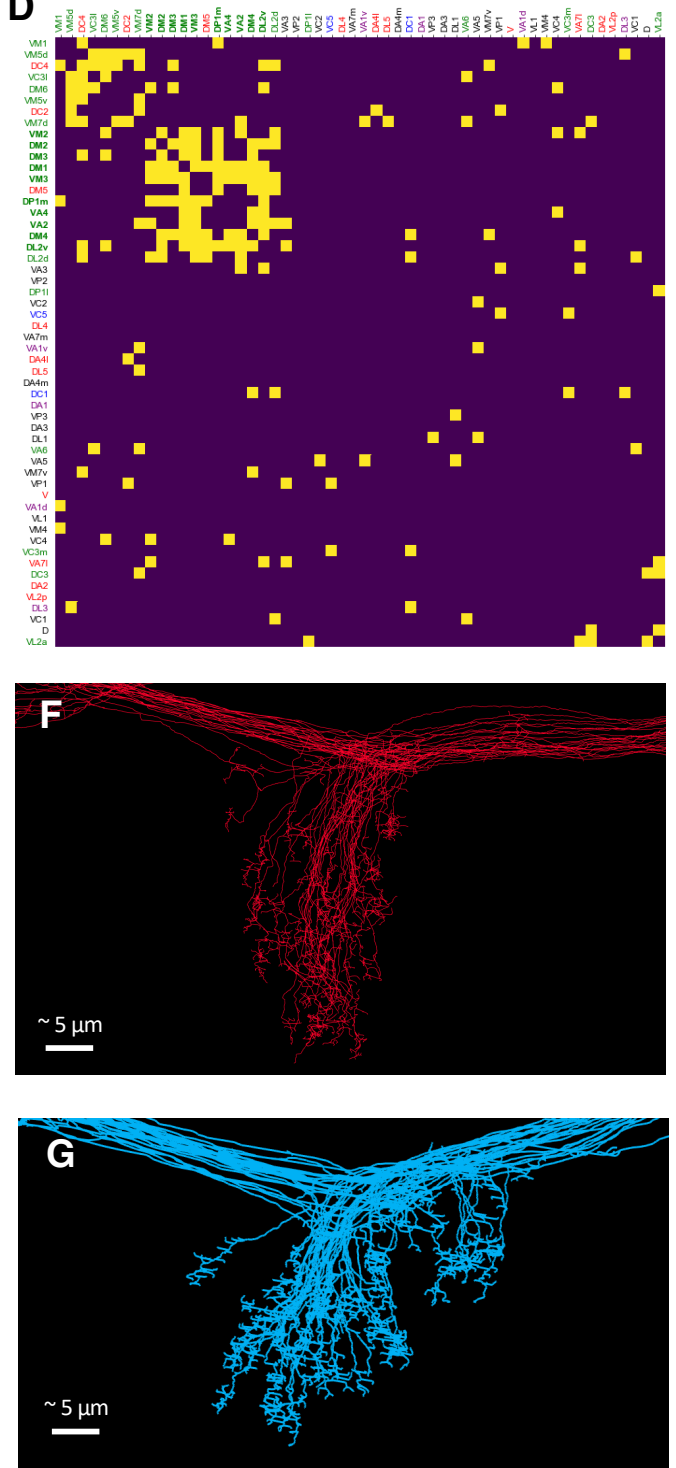
Supplemental Figure 5.
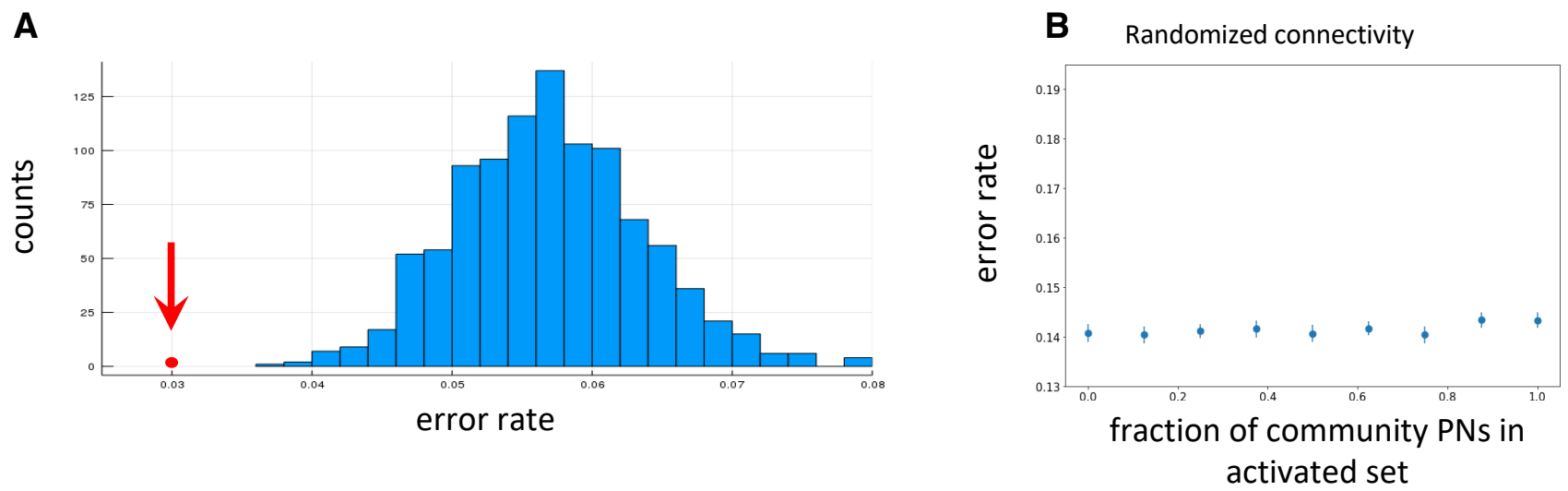


\section{Supplemental Figure 6. Random claw model}

A random claw model, randomized and reclustered

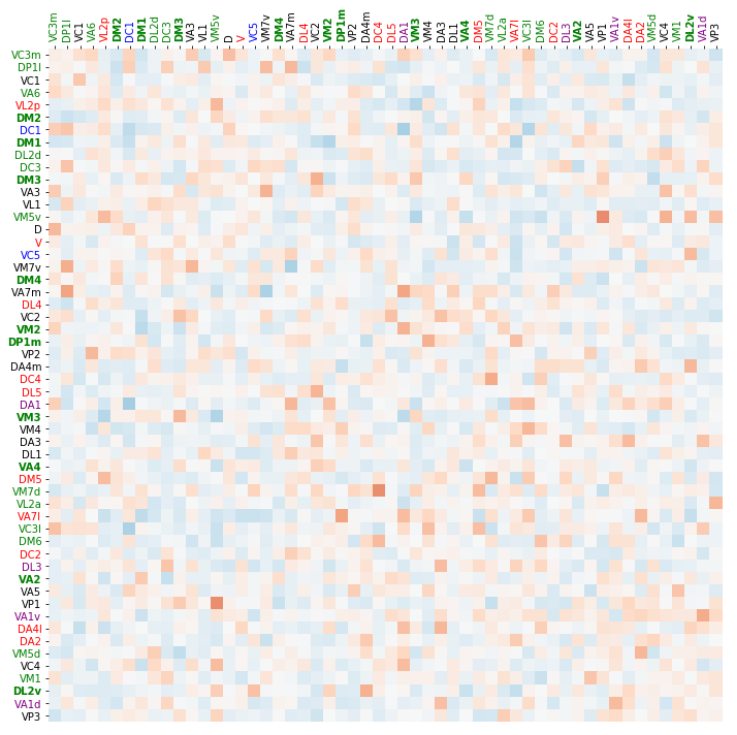

B random claw vs. random bouton models: z-score distribution

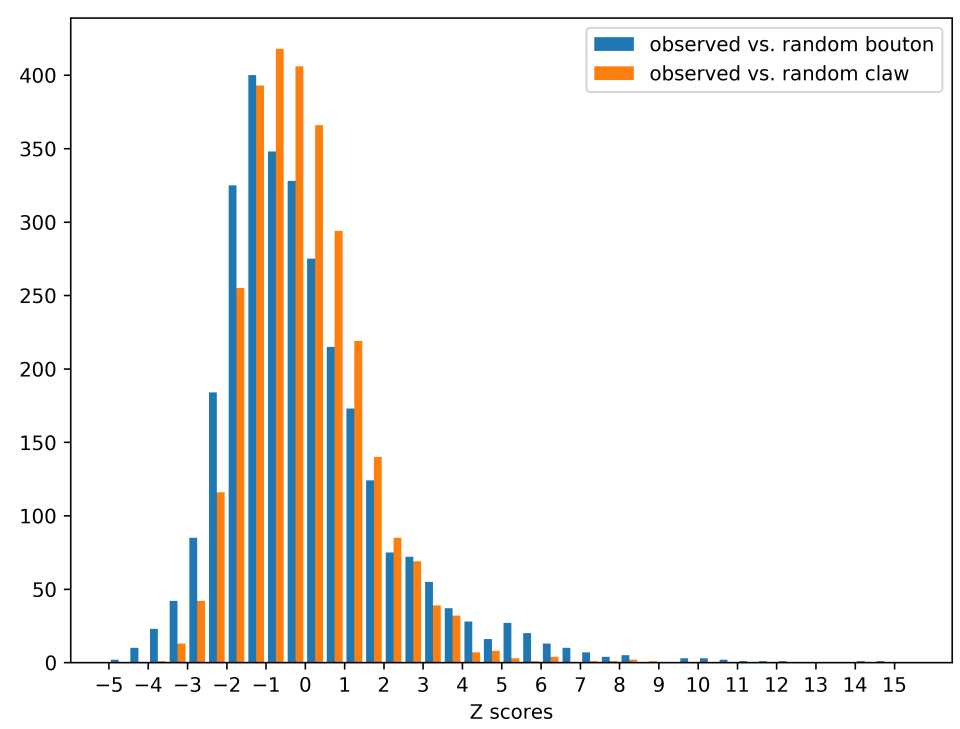


Supplemental Figure 7.

A Caron et al. data (random bouton null model)

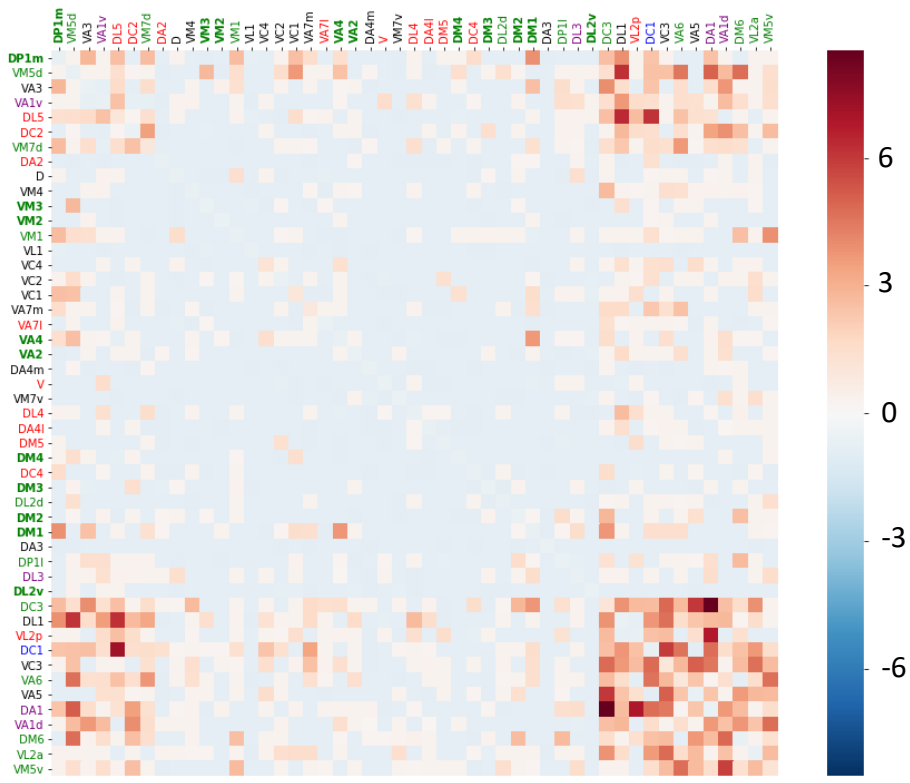

B

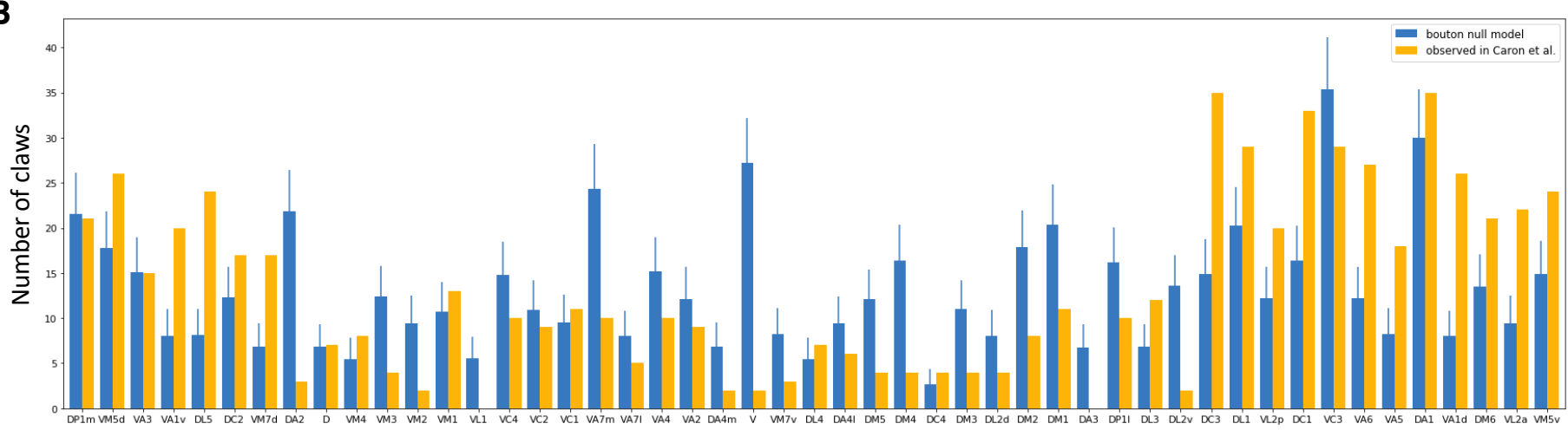

C

Caron et al. data (random claw null model)

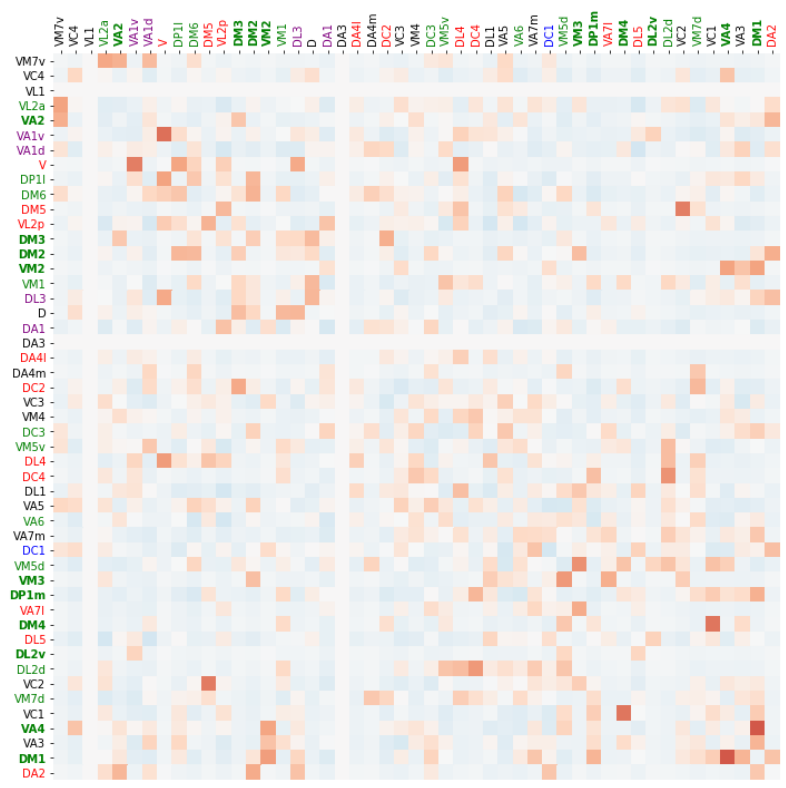

D Subsampling of data from current study, with Caron et al. sample size (random bouton null model)

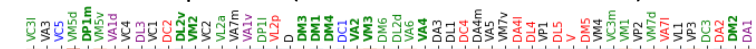

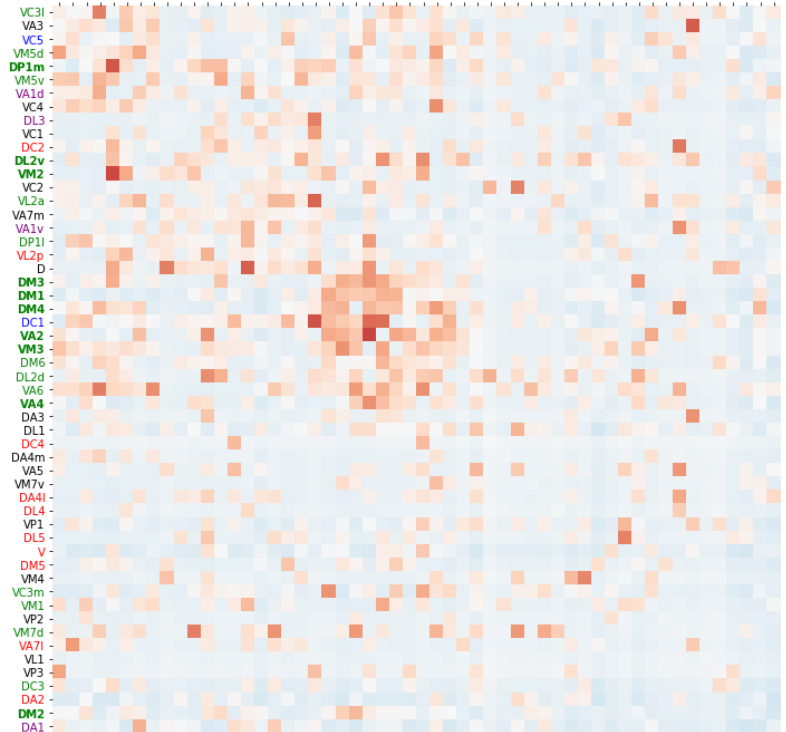


Supplemental Figure 8.
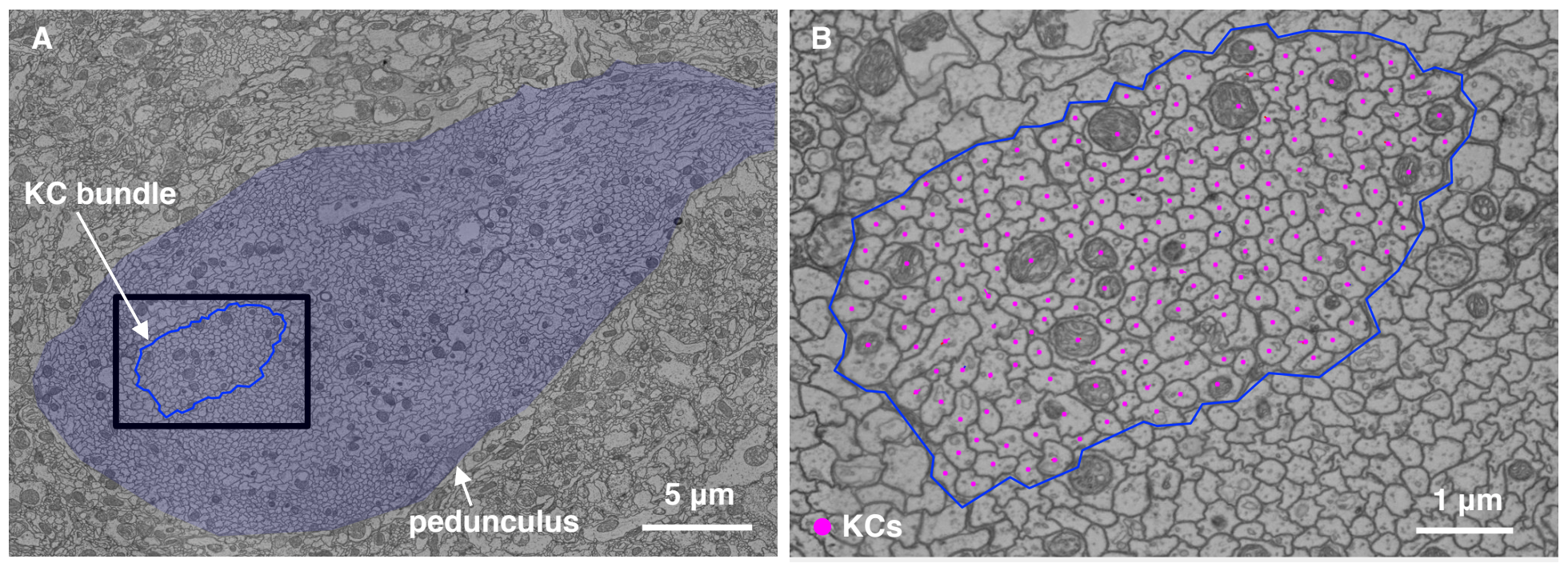
Supplemental Figure 9.

A

\section{Random}
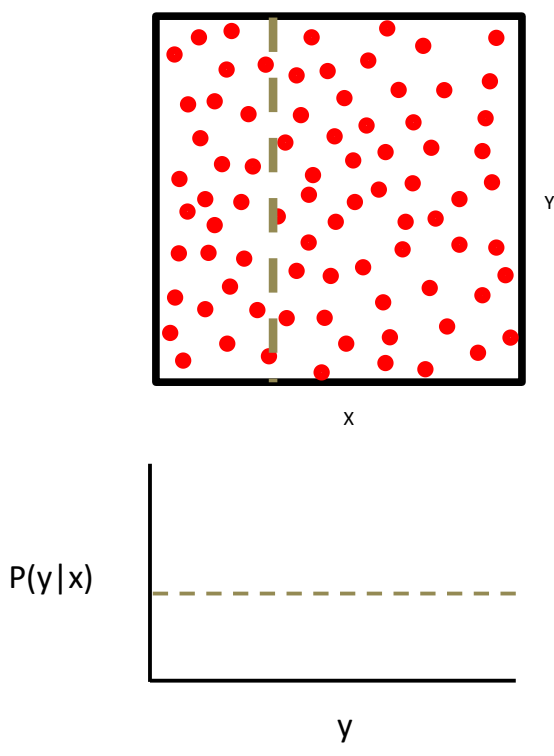

B

\section{'Associational fovea'}

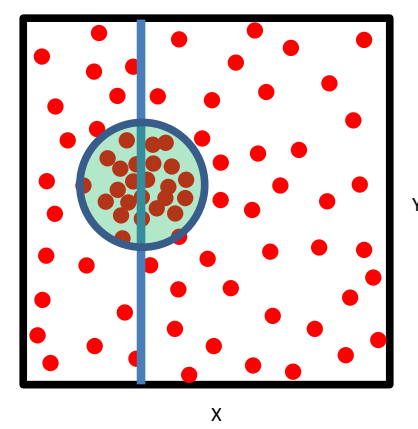

these combinations of odorants are presumably more frequent and more important-e.g. food
$P(y \mid x)$

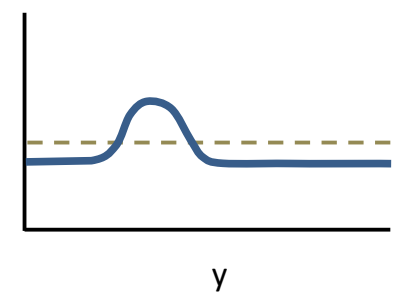

